\title{
A Tabu Search Based Metaheuristic for Dynamic Carpooling Optimization
}

\author{
Sondes BEN CHEIKH-GRAIET, Mariagrazia DOTOLI, and Slim HAMMADI
}

\begin{abstract}
The carpooling problem consists in matching a set of riders' requests with a set of drivers' offers by synchronizing their origins, destinations and time windows. The paper presents the so-called Dynamic Carpooling Optimization System (DyCOS), a system which supports the automatic and optimal ridematching process between users on very short notice or even en-route. Nowadays, there are numerous research contributions that revolve around the carpooling problem, notably in the dynamic context. However, the problem's high complex-ity and the real time aspect are still challenges to overcome when addressing dynamic carpooling. To counter these issues, DyCOS takes decisions using a novel Tabu Search based metaheuristic. The proposed algorithm employs an explicit memory system and several original searching strategies developed to make optimal deci-sions automatically. To increase users' satisfaction, the proposed metaheuristic approach manages the transfer process and includes the possibility to drop off the passenger at a given walking distance from his destination or at a transfer node. In addition, the detour concept is used as an original aspiration process, to avoid the entrapment by local solutions and improve the generated solution. For a rigorous assessment of generated so-lutions, while considering the importance and interaction among the optimization criteria, the algorithm adopts the Choquet integral operator as an aggregation approach. To measure the effectiveness of the proposed method, we develop a simulation environment based on actual carpooling demand data from the metropolitan area of Lille in the north of France.

Keywords: Dynamic Ridesharing, Multi-criterion Optimization, Tabu Search, Choquet Integral, Automatic ridematching.
\end{abstract}

\section{Introduction}

The average car occupancy decreased from 1.63 in 2000 to 1.57 in 2010, according to a study conducted by The Scottish Household Survey (3). In more recent years, USA have no better occupancy rates according to the latest National Household Travel Survey (2) and this has led to an increase in traffic congestion. Based on a study powered by INRIX Traffic Data, in 2011, congestion increased the urban travel time by over 5.5 billion hours, implying the consumption of an extra 2.9 billion gallons (59). To avoid the harmful impact of private cars and thus correct the negative environmental image associated with the private vehicle, carpooling (also called ridesharing) systems were born. The carpooling transportation system is based on a shared use of personal cars by a driver and one or more passengers, which can be casual or organized through a service (19) (47) (49). By making the private car a common mode of transport, carpooling plays a role in reducing the number of cars on the road and subsequently in limiting the greenhouse gas emissions. According to a recent study released in Tehran by (68), when all travellers choose carpooling, vehicle trips per day are reduced by 
780000 units and average annual fuel consumption is reduced by 336.53 million liters. Moreover, the concept of carpooling provides many advantages both at an individual and collective level (e.g. alleviating the household budget allocated to transportation, lightening road traffic, create social and cultural bonds, etc.).

This paper addresses the emerging need of automatic dynamic ridematching algorithms for smart mobility in metropolitan areas. Unlike traditional ridesharing, our Dynamic Carpooling Optimization System (DyCOS) proposes real time ridematching solutions and it does not require a long-term commitment between the rideshare partners. In other words, the routes' plan is drawn up dynamically and users accept to take part of a ridematching solution generated by the system.

The approach is based on a Tabu Search based metaheuristic and allows riders and drivers to make coordinated decisions taking into account five criteria related to time, economical and environmental aspects, while allowing transfers and detours. By using algorithms with a polynomial complexity, our resolution approach is implementable on different engineering software environment and is able to generate feasible solutions with an ICT tool for the smart mobility governance of a metropolitan area (23) (24) (12).

The literature review yields several research references aimed at providing efficient and optimized rideshar-ing services. Indeed, the design and development of advanced algorithms for associating optimally vehi-cle/passenger in real-time has received strong attention from the transportation community. However, to the best of our knowledge, no contribution exists in the related literature that integrates the multi-hop ridesharing with the detour and transfer concepts, including the possibility to drop the passenger off at a given walking dis-tance from his destination or a transfer node. To fill this gap, in this work we present the $D y C O S$ approach and report some computer simulations based on actual travel demand data from the multimodal transport network of the Lille metropolitan region (north of France) to test the performance of the proposed dynamic carpooling system. The main contributions of this study can be summarized as follows:

$\circ$ We develop a Multi-Criterion Metaheuristic Approach (MCMA) specifically adapted to the dynamic dimensioning of a system where new passengers and vehicles continuously enter and leave;

$\circ$ Thanks to the MCMA approach, we characterize the dynamic ridesharing by the detour and transfer concepts providing high quality solutions to practical DyCOS instances;

$\circ$ In addition to allowing travel costs savings and $\mathrm{CO}_{2}$ emission limitation, our dynamic carpooling system allows limiting the traffic congestion and thus decreasing system-wide travel times.

$\circ$ We adopt a realistic simulation scenario based on actual demand travel data from the Lille regional trans-port network and operate it to assess the performance of DyCOS. The simulation results measure the benefits of our advanced algorithms to satisfy as many users' requests as possible, while using the mini-mum number of resources (vehicles).

A detailed positioning of the paper with respect to the related literature while highlighting the novelty and merits of our approach is reported in the subsequent Section 2.3 We also note that this paper is an extended version of our previous papers that appeared as (15) and (13). Even if, in the work (13), we adopt the tabu search approach to solve the ridematching problem, it differs from this paper in several aspects. Indeed, in 
(13), we focus on the alliance between optimization and multi-agent system and we present a distributed multi-agent architecture where highly communicating entities evolve. Then, the optimization approach in (13) is only briefly introduced and not formalized, which is here fully accomplished. As regards the paper (15), in the present manuscript, we extend our previous work by: (A) performing an in-depth survey of existing carpooling systems, (B) studying the complexity of the dynamic carpooling problem, (C) illustrating in more depth the different steps of our optimization approach, (D) developing the theoretical aspects of our evaluation process where we apply a fuzzification method to normalize the values of the criteria and we adopt the Choquet Integral to take in consideration the interactions among them, (E) presenting new experimental results and highlighting the contribution of the Choquet Integral to ensure a rigorous evaluation of the generated solutions.

Moreover, in our previous related papers (17) and (16), we have proposed an evolutionary approach to man-age the real time multi-hop ridematching issue, where assignments are handled by an independent mechanism. By contrast, in the present Tabu Search based Methaheuristc, the assignments' process is a part of the global optimization algorithm and passengers are assigned on an as-needed.

The remainder of the paper is structured as follows: Section 2 presents briefly the state-of-the-art of the carpooling problem and positions our paper in this respect. In order to describe the framework of DyCOS, Section 3 outlines the complexity and the mathematical formulation of decision variables. Section 4 presents the MCMA approach developed to solve the ridematching problem. Section 5 shows some numerical results. Finally, Section 6 concludes the paper.

\section{State-of-the-art}

Over the past few years, we have witnessed the emergence of the ridesharing concept, thanks to its economi-cal and environmental benefits. This phenomenon is currently being reinforced by the multiplication of attempts to improve its services, leading the shared vehicle concept to exist in about 1000 cities around the world already (1). The effectiveness of the existing carpooling systems, however, is limited due to the lack of optimization and automation (60) (71).

According to Furuhata et al., design of attractive mechanisms, appropriate ride arrangement, and trust build-ing up among unknown travelers represent the major challenges to overcome to ensure service quality and cost control (26). Depending on the reservation management criteria adopted within the system, we distinguish be-tween two types of carpooling. The first one is planned in advance using a preliminary reservation, while the second one operates within a dynamic context. In the following, we present an overview of various carpooling attempts, while differentiating between: 1) the operational carpooling system, remaining typically open and non-optimized systems; 2) the academic studies implemented for designing and optimization of such systems.

We then discuss the use of metaheuristic and most notably of Tabu Search in the optimal transportation context. Finally, we position our paper with respect to the recalled literature, discussing its advantages and peculiarities. 


\subsection{Related works on ridesharing}

Also known as liftsharing, carpooling is released typically as online sites allowing users to consult and publish offers. Among the most popular platforms, we can mention Blablacar.fr in Europe (France, Italy, UK). However, this kind of platforms does not propose an automatic ridematching solution. The rider has to find his rideshare partners by checking the compatibility between the requests path and the proposed route, in order to be able to use a High Occupancy Vehicle (HOV) lane or to share the trip cost (11). However, due to their static reasoning, these systems demand advance booking independently of the uncertainties of road. To overcome this limitation, dynamic ridesharing systems provide an instantaneous localization and an accurate communication between users and the platform (eg. greenmonkeys.com, covivo.fr). This kind of carpooling is in the midst of a full expansion since it offers more flexibility than static approaches. The real challenges of these systems are: real time travel optimization and the guarantee of a reliable service (15).

From an academic standpoint, carpooling has entered the field of research and numerous approaches have emerged in the context of optimal ridesharing. The main objective of optimal ridesharing is to maximize the amount of matching between drivers and passengers or riders while optimizing several criteria such as travel time, cost, etc. From a modeling point of view, several optimal carpooling studies are applicable to the professional carpooling that ensures commuting from home to work. As an example, we might mention the tailored matching algorithm proposed by (51). Based on the Monte Carlo method, the algorithm manages the carpooling requests of a particular class of users (students, universities' employees, etc.). In their study, Calvo et al. present a carpooling platform dedicated to the employees of a company that using several instant data and communi-cation models for solving the Daily Carpooling Problem (58). Several suitable models are designed to address the many-to-many carpooling problems (from different origins to different destinations). According to Agatz et al., "these works mainly focus on matching between: (i) a single driver and multiple riders, (ii) multiple drivers and a single rider, (iii) multiple drivers and multiple riders" (6).

Otherwise, Son et al., claim that ridematching optimization is ensured by solving two NP-complete problems: managing the assignments passengers/vehicles and generating the shortest path for the drivers in order to reduce the trips cost. On the basis of this finding, the ridematching problem may be considered a multiobjective optimization problem with high complexity. Therefore, to solve these models, various heuristic or agent-based simulation methods have been developed. In their study, Shete et al. propose a metaheuristic called genetic-based carpool route and matching algorithm to solve the multi-objective carpool service problem (62). Son et al. also consider a multi-objective dynamic carpooling problem that minimizes the trips cost and optimizes the routes time. The authors adopt labeling algorithms to solve the multi-objective shortest path problem (67). To tackle the problem complexity, Manel et al. propose in their research a subdivision principle to decompose the carpooling network and consider a distributed dynamic graph (60). Then, authors adopt the multi-agent concept to establish a distributed parallel process (61). While the emphasis lies in finding the best matching between riders' requests and drivers' offers, Czioska et al., focused on the assessment scheme for meeting point locations(21). In fact, according to the authors: "the usage of meeting points can be beneficial since the drivers do not have to pick up the passengers at the doorstep and drive unnecessary detours".

Integrating new technologies such as GPS-enabled mobile devices and the ubiquitous wireless networks, 
Chung-Min et al. develop a smart ride-share system with a scheduling algorithm that combines a greedy method and the bi-partite matching algorithm to solve ridematching problem (18). Through the new communication technologies, several recent studies attempt to match drivers and riders in a very dynamic context. In this sence, Lalos et al. develop a framework using positioning systems to support a dynamic network of car and taxi pool services (44). The adaptation of algorithms from a static context to a dynamic context has often been adopted, but most studies ignore the need for optimizing the users' collaboration through a communication architecture (see, e.g., (34) and (45)). In fact, an efficient communication between users is crucial for setting up a proficient dynamic carpooling system. It is in this sense that Nagare et al. use the Android platform to develop the so-called Enable Dynamic Carpooling application which implements an efficient communication between car owner and ride seeker (54). Trying to take advantages of the Information and Communication Tech-nologies (ICT $s$ ) in developing intelligent transportation systems, Dimitrakopoulos et al. also propose a novel management functionality for dynamic carpooling called $i$ CAP. The system aims at exploiting knowledge and experience from past interactions, in proposing reliable matches between drivers and riders (passengers) (22).

By authorizing the transfer, a rider can be transported by more than one vehicle to reach his final destination. This kind of ridesharing is called Multi-hop Ridesharing $(M H R)$ and it is obviously more flexible than other forms of carpooling and offers more options for users. In his research, Gruebele (57) argues that a driver and a passenger should not be matched only if they share the same or similar destination because perfect matching would claim high waiting times. Therefore, the author proposes a dynamic multi-hop ridesharing system, by dividing a passenger route into smaller segments as parts of other trips. According to Herbawi et al., in a

M $H R$ problem, the set of drivers' offers that constitute a rider's trips are called the route plan, which is subject to multi-objective optimization such as the minimization of time and cost (70). To solve this problem, authors model the route planning in the dynamic $M H R$ as the shortest multi-objective path problem on a time-expanded graph representing the drivers' offers. Then, they propose an evolutionary solution approach, which is able to provide a good quality set of route plans and outperforms the generalized label correcting algorithm

in terms of computing time. However, in their version of the $M H R$ problem, drivers do not deviate from their routes and time schedules, which may reduce flexibility in generating solutions. The routing of drivers is considered as an interesting area of research related to multi-hop passenger transportation systems. Instead of focusing only on efficiently routing passengers based on a given network with transfer nodes, Agatz et al. emphasize that where to locate the pick-up or transfer locations could be an interesting issue (6). In this context, some studies introduce the detour concept, where a driver agrees to change his itinerary to pick-up a passenger from his origin or even agree to a transfer point that does not belong to his route. Geisberger et al. (27) develop an algorithmic solution to efficiently compute detours to match ridesharing offers and requests. On condition that the length of the resultant route does not exceed a predefined threshold, the authors propose to allow the driver to slightly modify his itinerary to serve more riders. 
Regarding pricing, the literature on ridesharing considers different travel costs that are roughly proportional to vehicle-miles (5). Other ways to divide the trip costs between the ridesharing partners are suggested. An auction-based mechanism to calculate the drivers' reimbursement is presented by Kleiner et al. (42). Indeed, each passenger has to pay the difference between the cost of driving alone in a private car and the cost of taking a taxi. In their study, Ferreira et al. propose an architecture for a collaborative carpooling system based on a credits mechanism to encourage the cooperation among riders and drivers (36). Then, the accumulated credits can be converted into parking facilities.

Summing up, while there are some attempts to provide dynamic carpooling service, only few have met the expected success, mainly because of the lack of flexibility and incentives (26). Graziotin et al. show how dynamic carpooling systems still have numerous important open issues to be addressed and solved, such as reaching the critical mass, which necessarily refers to issues as incentives, safety and trustiness (29). That explains the current absence of any dynamic carpooling system deployed and used for real life (29).

In order to overcome some limitations of the discussed existing services, such as optimization and dynamic aspects, in this paper we present a Dynamic Carpooling Optimization System (DyCOS) which manages in real time a set of offers and requests and proposes optimized ridematching according to a set of criteria and within certain constraints. The key contribution of this work is to satisfy the maximum number of requests using a minimum number of carpooling vehicles thanks to the authorization of the transfer process. As regards the optimization approach, we develop a multi-criterion Tabu Search technique that minimizes users' waiting time, delay and trip's cost, and maximizes the gain in terms of $\mathrm{CO}_{2}$. In addition, the integration of the detour concept as an aspiration criterion for the tabu approach allows to increase the number of served requests, while using the same resources (vehicles).

\subsection{Metaheuristic and Tabu Search for optimal transportation problems}

Blum et al. state that approximate methods do not seek to find optimal solutions, but rather good solutions within a reduced calculation time (10). By exploring and exploiting the search space, metaheuristic are able to find efficiently near-optimal solutions (56) (40)(37). In the literature, several metaheuristic are distinguished, such as Ant Colony Optimization, Evolutionary Computation including Genetic Algorithms, and Tabu Search (39).

In computer science, metaheuristic such as evolutionary algorithms and Tabu Search are regarded as "anytime algorithms" that can generate a feasible solution at any time. In other words, such an algorithm is designed to optimize the generated solutions as work has progressed (38)(41). Operating in a dynamic context, the dy-namic carpooling system has to deal with several instantaneous events and make quick decisions (46). Starting from this observation, metaheuristic based on anytime algorithms are particularly well suited to solve these kinds of issues. For this reason, Tabu Search can be seen as a valid optimization method

to solve optimal ridesharing problems in a dynamic setting. The main principle of such a method consists in examining successive neighbors of a solution, and the objective is allowed to deteriorate in order to avoid local minima (48).

In the area of transportation research, Tabu Search is widely used for solving routing problems (35). Nanry et al. develop a reactive tabu search approach to solve the pickup and delivery problem with time windows using different move neighborhoods (55). Cordeau et al. describe a tabu search heuristic to solve the 
dial-a-ride problem with multiple vehicles. The proposed approach is characterized by a procedure for neighbourhood evaluation that adjusts the visit time of the vertices on the routes so as to minimize route duration and ride times (20).

In the carpooling domain, very few studies adopt the Tabu Search approach to solve the ridematching prob-lem. In their research, Wang et al. propose an insertion heuristics to construct the initial routes based on random assignments. Then, they use a Tabu Search algorithm to improve the generated solution (69). In the dynamic context, Berbeglia et al. develop a hybrid algorithm that combines an exact constraint programming algorithm and a tabu search heuristic (9). Generally, the computational results of these approaches demonstrate the efficiency, effectiveness, and scalability of the Tabu Search algorithm.

In the same context, we can mention the Tabu search algorithm with four neighborhood structures proposed by Can et al., (33) to solve a variant of the carpooling problem. Based on a two-stage integer programing, the TS algorithm transforms the neighborhood structure suitable for vehicle routing problem VRP into four types of neighborhood. Although initial computational results are encouraging, the proposed approach cannot manage time window constraints, indicating a notable gap.

\subsection{Positioning of our work}

To summarize, the originality of our approach lies in the fact that:

1. The proposed $D y C O S$ manages the many-to-many ridematching problem, contrary to carpooling systems developed by Bruglieri et al. in (9) or set up by Son et al. in (67) which manage only the homework trips. Moreover, our approach generates ridesharing solutions based on the similarities between the routes proposed by drivers and the trips requested by riders. This differs from taxis-based systems where the drivers do not impose travel restrictions (72) (7).

2. The proposed system integrates the multi-hop ridesharing with detour and transfer concepts. This is deemed to be the main distinction between our paper and the previously recalled literature, particularly (44) and (67), where each request is served by a single vehicle and routes' deviation is not tolerated. We remark that, even if the detour concept is considered in some studies (e.g. (27) and (69)), they ignore the transfer process for reasons of simplification. For greater comfort, our system seeks initially to propose ridesharing solutions without transfer.

3. Although Tabu Search has been adopted in (33) to solve the ridesharing problem, the proposed algorithm addresses the variant model of the long-term carpooling problem which involves multiple origins and one destination. By contrast, our DyCOS proposes carpooling solutions from multiple origins to multiple destinations.

4. Unlike classical approaches, where a request is served only if it reaches its final destination (60), our system includes the possibility of dropping off the passenger at a maximum walking distance from his final destination or transfer node. This increases flexibility and enhances the service quality.

5. In contrast to the classic Tabu Search algorithms developed in (71), our optimization algorithm (MCMA) adopts the detour concept as an original aspiration criterion. 
6. To avoid degradation of the generated solutions, aspiration is authorized only if the detour is less than a Tolerated Detour Distance $(T D D)$ specified by the system.

7. We consider a multi-criterion dynamic carpooling problem, where the travel time, the cost and the $\mathrm{CO}_{2}$ emission are to be minimized. To the best of our knowledge, there exists no previous study that considers the environmental gain in term of $\mathrm{CO}_{2}$ of the carpooling practice. In the majority of cases, researchers seek to minimize travel time and trips cost while maximizing the number of served requests (e.g. as in (70), (69), (5) and (67)).

8. The idea of using the Choquet integral as an aggregate approach promotes a compensation aspect between criteria accelerating the convergence of the proposed MCMA.

9. $M C M A$ is able to effectively manage the detour and transfer concepts, allowing us to find good solutions using a minimum number of vehicles to serve a maximum number of riders.

3. The optimal dynamic carpooling problem statement

\subsection{System description}

The deployment of a carpooling service should be supported by multiple features. In view of the complexity of the operations, the idea of the subdivision of the system into sub-models has emerged. Each sub-model is dedicated to a specific macro-function. In this context, our system consists of several fonctionalities principally concerned with the request acquisition, the assignment process, and the evaluation process.

In order to measure the ridematching problem's complexity and deal with such difficulties, we carry out a comparative study between the carpooling problem and other optimization problems known for their complex-ities.

The Traveling Salesman Problem (TSP) is a well-recognised problem known by its exponential complexity (52). Given a list of cities and their pairwise distances, the problem is to find the shortest path for the sales-man while visiting each city, only once. Instead, the Dynamic Carpooling Problem (DCP) consists in bringing together people with similar itineraries and time schedules to share rides on short-notice. Then, slightly modi-fied, the $T S P$ appears as a sub-problem in the $D C P$. Indeed, in a simple instance of the $D C P$, where one vehicle respecting the $T S P$ constraints carpools several passengers (riders), the concept of salesman represents the vehi-cle, the concept of city represents origins and destinations of passengers, and the concept of distance represents traveling times or cost (Figure 1). This paper treats the DCP of matching drivers (more than one vehicle) and passengers in this dynamic setting. Therefore, we can consider our dynamic carpooling optimization problem as a Multi-TSP (Figure 2).

We finally remark that, in the DyCOS application, additional constraints such as limited resources (vehicles' capacity) or time windows can further accentuate the complexity of the problem.

\subsection{Mathematical formulation}

Some explanations about the used parameters and variables are listed in Table 1 and their specific meaning will also be discussed in the text. 


\begin{tabular}{cc}
$P^{l}:$ the passenger $l$ & $V^{k}:$ the vehicle $k$ \\
$R_{p}(t):$ the set of $n d$ instantaneous requests & $O_{V}(\mathrm{t}):$ the set of $m$ vehicles offers \\
$P^{l} \quad$ : origin of passenger $P^{l}$ & $P^{l+}:$ destination f passenger $P^{l}$ \\
$V^{k} \quad:$ origin of vehicle $V^{k}$ & $V^{k+}:$ destination of vehicle $V^{k}$ \\
$Q^{l} \quad 1:$ number of places requested & $L^{k}: V^{k}$ s capacity \\
$d^{l}:$ the earliest departure time of passenger $P^{l}$ & $a^{l}:$ the latest arrival time of passenger $P^{l}$ \\
$D_{i}^{k}$ : the estimated departure time of vehicle $V^{k}$ from point $i$ & $A_{i}^{k}:$ the estimated arrival time of vehicle $V^{k}$ to point $i$ \\
\hline
\end{tabular}

\subsubsection{Decision variables and parameters}

We denote $R_{p}(\mathrm{t})=\left\{R_{l}, R_{2}, \ldots R_{l}, \ldots, R_{n_{r}}\right\}$ the set of $n$ instantaneous demands received during a short interval of time $\Delta t$ for $P=\bigcup_{l=1}^{n}\left\{P^{l}\right\}$ which is the set of $n$ passengers with $\left(n_{r} \leq n\right)$. Equation (1) shows passenger $P^{l}$ request's formulation:

$$
R_{l}(t)=\left(P^{l-}, P^{l+}, d^{l}, a^{l}, Q^{l}\right)
$$

Where moving preferences are specified:

○ $P^{l}, \mathrm{Pl}^{+}$are respectively the current position (the origin) and the destination node asked by the passenger $P$,

○ $d^{l}, a^{l}$ indicate the earliest departure time and the latest arrival time preferred by $P^{l}$,

○ $\quad Q^{l} 1$ is the number of passengers including $P^{l}$ who wish to travel together. In other words, if a group of people (friends, employees..) have the same origin and destination and they want to travel in the same time windows, they will send one request where they specify the number of passengers $Q^{l}$.

Similarly, we denote by $O_{V}(t)=\left\{O_{V^{1}}, O_{V^{2}}, \ldots, O_{V^{k}}, \ldots, O_{V^{m}}\right\}$ the set of $m$ vehicles offers received at time $t$, where $V=\bigcup_{k=1}^{m}\left\{V^{k}\right\}$ is the set of $m$ vehicles expressing travel deals. Equation (2) shows the formulation of vehicle $V^{k}$ 's offer:

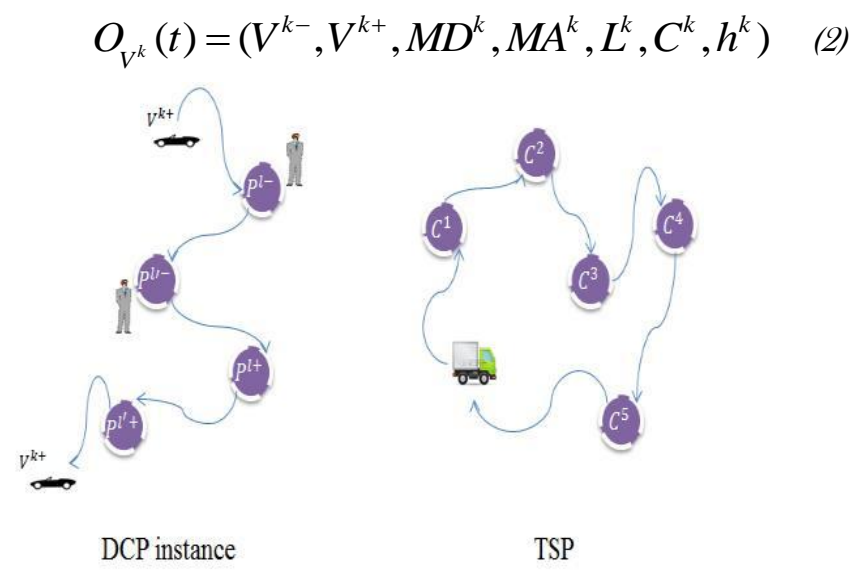

Figure 1: Analogy between the dynamic carpooling problem and the travelling salesman problem. 


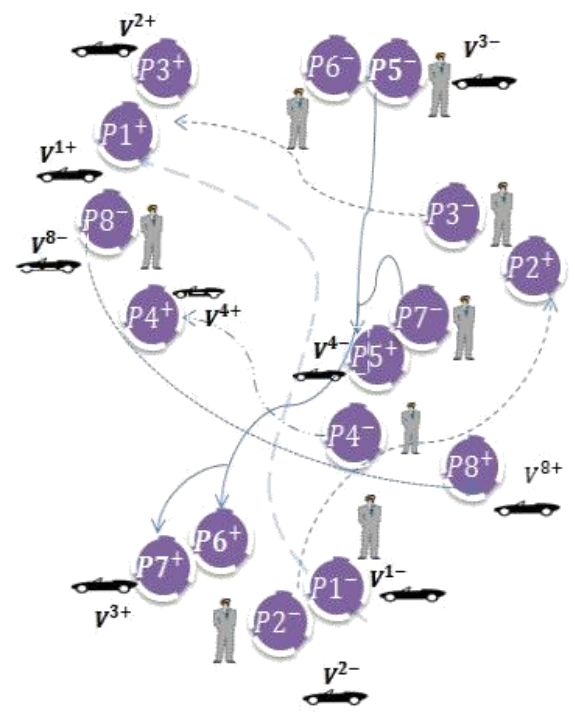

Figure 2: Analogy between the dynamic carpooling problem and the multi-travelling salesman problem.

Where $V^{k}, V^{k+}$ refer respectively to the origin and the destination of the vehicle $V^{k}$ with a capacity $L^{k}$. $M D^{k}, M A^{k}$ indicate respectively the vector of the estimated departure time denoted $D_{i}^{k}$, and the vector of the estimated arrival time denoted $A_{i}^{k}$ of the vehicle $V^{k}$ on all nodes $i$ between $V^{k}$ and $V^{k+}$. According to the traffic condition, these nodes correspond to the intermediate destinations specified by the driver in real-time. Moreover, each vehicle $V^{k}$ is characterized by a kilometric cost criterion that we denote $C^{k}$ and a criterion of emission rate of $\mathrm{CO}_{2}$ per kilometer denoted $h^{k}$.

\subsubsection{Criteria}

\subsubsection{The total waiting time}

This criterion aims to minimize the customers' waiting time in the departure point and in the transfer node. Therefore, for each passenger $P^{l}$ assigned first to $V^{k}$ then to $V^{k^{\prime}}$, the waiting time in origin (i= $P^{l-}$ ) is the difference between the departure time desired by $P^{l}$ and the departure time of $V^{k}$ Then, the waiting time in the transfer node corresponds to the difference between the departure time of $V^{k^{\prime}}$ and the arriv (1)al time of $V^{k}$ in the transfer node j. Subsequently, the $P^{l}$ waiting time is formulated as:

$$
W T_{l k k^{\prime}}=\operatorname{Max}\left(0,\left(D_{i}^{k}-d^{l}\right) * X_{l k}+\left(D_{j}^{k^{\prime}}-A_{j}^{k}\right) * X_{l k^{\prime}}\right)
$$

Where $X_{l k}$ is a decision variable which is equal to 1 if $P^{l}$ is affected to $V^{k}, 0$ otherwise.

Then, The total passengers waiting time at the different nodes is equal to:

$$
T W T=\sum_{\substack{k=1 \\ k^{\prime}=1 \\ k^{\prime} \neq k}}^{m} \sum_{l=1}^{n} W T_{l k k^{\prime}}
$$

\subsubsection{The total delay time}


This criterion seeks to minimize the delay time which corresponds to the difference between the desired arrival time specified by the passenger and the actual arrival time to its final destination $\left(\mathrm{i}=P^{l+}\right.$ ). It can be defined as follows:

$$
W T_{l k k^{\prime}}=\operatorname{Max}\left(0,\left(A_{i}^{k}-a^{l}\right)\right)
$$

Consequently, the total delay time is calculated as:

$$
T D T=\sum_{k=1}^{m} \sum_{l=1}^{n} D T_{l k} * X_{l k}
$$

\subsubsection{The total route time}

The route time criterion consists of minimizing the total journey times aboard the various vehicles. For each passenger $P^{l}$ assigned to $V^{k}$ from i to j, the route time is stated as follows:

$$
R T_{l k}=\left(A_{j}^{k}-D_{i}^{k}\right) * X_{l k}(7)
$$

We suppose that $P^{l}$ was assigned to $V^{k}$ at the departure point and to $V^{k^{\prime}}$ at the transit point. Then the global route time is formulated as:

$$
R T_{l k k^{\prime}}=R T_{l k}+R T_{l k^{\prime}}
$$

Subsequently, to evaluate the solution, we need the total route time of all passengers which is determined as:

$$
T R T=\sum_{k=1}^{m} \sum_{\substack{k^{\prime}=1 \\ k^{\prime} \neq k}}^{m} \sum_{l=1}^{n} R T_{l k k^{\prime}}
$$

\subsubsection{Environmental criterion}

This criterion determines the improvement in terms of $\mathrm{CO}_{2}$ established in both of the following cases:

- -Passenger uses his own vehicle,

- -Passenger chooses to carpool.

3.2.2.4.1. $\mathrm{CO}_{2}$ emission quota without carpooling:

$$
I C_{l k_{l}}=h^{k_{l}} * \operatorname{distnace}\left(P^{l+}, P^{l-}\right)
$$

Where $I C_{l k_{l}}$ is the emission quota of $C O_{2}$ if the passenger $P^{l}$ uses his own vehicle denoted $V^{k_{l}}$ which is characterized by $h^{k_{l}}$, its emission rate of $\mathrm{CO}_{2}$ per kilometer.

3.2.2.4.2. $\mathrm{CO}_{2}$ emission quota with carpooling:

$$
S C_{l k}=\frac{h^{k} * R T_{l k} * \text { AverageSpeed }}{\text { number_of _ passenger } \mathrm{s}_{-} \mathrm{i} n_{-} V^{k}}
$$

Where $S C_{l k}$ is the emission quota of $\mathrm{CO}_{2}$ for each passenger sharing the same vehicle $V^{k}$ which is characterized by $h^{k}$. Hence, the gain of $\mathrm{CO}_{2}$ per passenger is calculated as follows:

- Without transfer:

$$
\operatorname{Gain}_{l k}=I C_{l k_{l}}-\left(S C_{l k} * X_{l k}\right)
$$

- With transfer: 


$$
S C_{l k k^{\prime}}=S C_{l k} * X_{l k}+S C_{l k^{\prime}} * X_{l k^{\prime}}
$$

So,

$$
\operatorname{Gain}_{l k k^{\prime}}=I C_{l k_{l}}-S C_{l k k^{\prime}}
$$

In that case, the total environmental gain realized by all passengers is determined by:

$$
T E G=\sum_{k=1}^{m} \sum_{\substack{k^{\prime}=1 \\ k^{\prime} \neq k}}^{m} \sum_{l=1}^{n} \text { Gain }_{l k k^{\prime}}
$$

\subsubsection{The trip cost}

According to the cost per kilometer $C^{k}$ of each vehicle $V^{k}$, this criterion seeks to minimize the trip cost which is formulated as follows:

- Without transfer:

$$
C K_{l k}=C^{k} * R T_{l k} * \text { AverageSpeed }
$$

- With transfer:

$$
C K_{l k k^{\prime}}=C K_{l k} * X_{l k}+C K_{l k^{\prime}} * X_{l k^{\prime}}
$$

Then, the total cost for all passengers is calculated by:

$$
T C=\sum_{k=1}^{m} \sum_{\substack{k^{\prime}=1 \\ k^{\prime} \neq k}}^{m} \sum_{l=1}^{n} C K_{l k k^{\prime}}
$$

Thereafter, the aggregation criteria process is determined by applying the Choquet Integral taking into account the weighting, the interaction and the compensation between different criteria

\subsubsection{Constraints}

Several constraints have to be considered during the real time assignment of passenger $P^{l}$ to vehicle $V^{k}$. We take into account the following capacity constraints:

$$
1 \leq Q^{l} \leq L_{i}^{k} \leq L^{k}
$$

Where $L_{i}^{k}$ is the residual capacity of $V^{k}$ on node $i . V^{k}$ must have enough available seats in node $i$ according to the number of seats asked by the passenger $P^{l}$. We also consider the following time constraint:

$$
d^{l} \leq D_{i}^{k}=P l
$$

In other words, $P^{l}$ can be assigned to $V^{k}$ only if his departure time at the earliest $d^{l}$ from his origin $\mathrm{P}^{1-}$ is earlier than or equal to the departure time specified by the driver.

Finally, another time constraint is:

$$
A_{i}^{k} \leq D_{i}^{k^{\prime}}
$$

In fact, in the case of a transfer, $V^{k}$ must reach the transfer node $i$ at the latest before the departure time of $V^{k^{\prime}}$. 
We conclude this section with a remark on the $D C P$ computational complexity. In the theory of computational complexity, the described problem is considered as NP-complete problem, implying that the computational time increases exponentially with the number of riders to serve and the fleet of vehicles to manage. As a result, solving the $D C P$ is typically highly challenging for systematic optimization methods, notably exact ridematching approaches that integrate the process of the transfer and detour. Therefore, this problem is more efficiently solved using heuristic methods or variants of local search approaches(6). To this aim, we propose a metaheuristic approach based on Tabu Search. It is a popular method, which its performance is proven by its many applications to effectively solve combinatorial optimization problems such as job-shop scheduling in (63), facility layout problems relating to the manufacturing in (73), traveling salesman problems in (25), and vehicle routing problem and its variants in (8) and (43).

\section{The proposed Tabu Search based metaheuristic}

As previously discussed, the dynamic carpooling problem including detour and transfer is an NP-hard prob-lem and the application of approximate methods to solve it has attracted an increased attention. Motivated by the success of the Tabu Search approach to solve the ridematching problem, we describe here a new Tabu Search procedure for the multi-criterion dynamic carpooling. It differs from the approaches presented in Section 2 in several aspects. In fact, unlike other applications, our Tabu algorithm starts by building a feasible initial solution that takes into account the similarity between users' routes and does not violate the capacity constraint. This allows improving the performance of the Tabu method and accelerating the convergence of our algorithm with respect to those proposed in (69) and (9). Moreover, in order to improve the solutions' quality, our Tabu Search algorithm is characterized by an original aspiration criterion. This latter allows 'excellent' Tabu Moves to be selected if the aspiration level is attained, which avoids the entrapment by local solutions. To carry out a rigor-ous assessment of the obtained solutions, our Tabu Search algorithm includes the Choquet Integral operator that takes into consideration the interactions among criteria. Finally, in order to deal with the high complexity of our problem, we decompose our global tabu search algorithm into several sub-algorithms that run sequentially.

We have already briefly introduced the idea underlying this method in (15). In this paper, we formalize and detail the different aspects of our metaheuristic and illustrate the different steps to solve the dynamic carpooling problem.

\subsection{Solution generation: Assignment Process}

In this study, we propose a Multi-criterion Tabu Search Algorithm (MTSA) for the DyCOS. The starting point is an initial solution $s_{0}$, usually randomly generated. Then, the algorithm explores the neighborhood to find a better solution, denoted $s_{t}$. To avoid cycling, the algorithm considers the recently visited solutions as tabus.

\subsubsection{Data modeling}

This process involves establishing the matrix of possible assignments Vehicle / Passenger in origins and in transfer nodes, with the aim of ensuring users comfort. In other words, the assignment is establish only if the 
distance between the rider's origin $P^{l}$ and the current position of the vehicle $V^{k}$ does not exceed the radius $R$. The latter is a parameter to define by the passenger and it presents the maximum tolerated walking distance. Similarly, in a transfer case, the rider $P^{l}$ is assigned to the vehicle $V^{k^{\prime}}$ only if the distance between the deposit node and the final destination $P^{l+}$ is less thane $R$ (Figure 3). Within this framework, our system generates a matrix $D V M$ (Departure Vehicle Matrix) that represents all possible assignments Vehicle/Passenger in the origin nodes of passengers. The assignment of the generic element of $D V M$ is as follows.

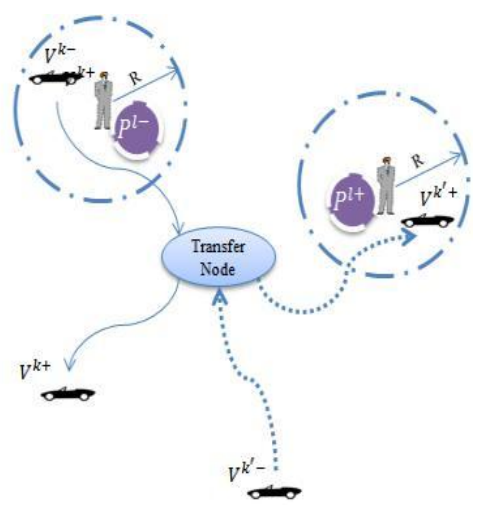

Figure 3: The possible assignment of passenger $P^{l}$ to vehicle $V^{k}$ and $V^{k^{\prime}}$

Table 2: Matrix $D V M$ of possible assignments vehicle / passenger in departure nodes

\begin{tabular}{|c|c|c|c|c|c|c|c|}
\hline$P^{l} / V^{k}$ & $V^{1}$ & $V^{2}$ & & $V^{k}$ & & $V^{m} 1$ & $V^{m}$ \\
\hline$P^{1}$ & $*$ & $\mathrm{X}$ & & $\mathrm{X}$ & & $*$ & $*$ \\
\hline$P^{2}$ & $\mathrm{X}$ & $*$ & & $*$ & & $\mathrm{X}$ & $*$ \\
\hline$\vdots$ & $\vdots$ & $\vdots$ & & $\vdots$ & & $\vdots$ & $\vdots$ \\
$P^{l}$ & $\cdot$ & $\cdot$ & & $\cdot$ & & $\cdot$ & $\cdot$ \\
$\vdots$ & $:$ & $:$ & & $\vdots$ & & $\vdots$ & $\vdots$ \\
\hline$P^{n} 1$ & $\mathrm{X}$ & $*$ & & $\mathrm{X}$ & & $*$ & $\mathrm{X}$ \\
\hline$P^{n}$ & $*$ & $\mathrm{X}$ & & $*$ & & $\mathrm{X}$ & $\mathrm{X}$ \\
\hline
\end{tabular}

Table 3: Matrix TV $M$ of possible assignments vehicle / passenger in transfer nodes

\begin{tabular}{|c|c|c|c|c|c|c|c|}
\hline$P^{l} / V^{k}$ & $V^{1}$ & $V^{2}$ & & $V^{k}$ & & $V m 1$ & $V^{m}$ \\
\hline$P 1$ & $\mathrm{X}$ & $*$ & & $\mathrm{X}$ & & $*$ & $*$ \\
\hline$P^{2}$ & $*$ & $\mathrm{X}$ & & $\mathrm{X}$ & & $\mathrm{X}$ & $*$ \\
\hline$\vdots$ & $\vdots$ & $\vdots$ & & $\vdots$ & & $\vdots$ & $\vdots$ \\
$P^{l}$ & $\mathrm{X}$ & $*$ & & $\mathrm{X}$ & $\vdots$ & $*$ & $\mathrm{X}$ \\
$\vdots$ & $\vdots$ & $\vdots$ & & $\vdots$ & $\vdots$ & $\vdots$ & $\vdots$ \\
\hline$P^{n} 1$ & $*$ & $*$ & & $\mathrm{X}$ & & $*$ & $\mathrm{X}$ \\
\hline$P^{n}$ & $\mathrm{X}$ & $\mathrm{X}$ & & $*$ & & $*$ & $\mathrm{X}$ \\
\hline
\end{tabular}


If (distance $\left.\left(P^{l} ; V^{k}\right) \leq R\right)$ and (respecting the capacity constraint (19)) Then $D V M\left[P^{l} ; V^{k}\right]=*$. Else $D V M\left[P^{l} ; V^{k}\right]=X$ (see the example in Table 2$)$.

Likewise, a matrix TVM (Transit Vehicle Matrix) is generated representing all possible assignments Vehi-cle/Passenger in the destination nodes of passengers: If $\left.\operatorname{distance}\left(P^{l+} ; V^{k}\right) \leq R\right)$ Then $T V M\left[P^{l} ; V^{k}\right]=*$. Else, $T V M\left[P^{l} ; V^{k}\right]=X$ (see the example in Table 3$)$.

We also define the matrix INV (Interconnected Nodes between Vehicles) which presents all intermediate destinations of vehicles. We note $N=\mathrm{U}_{i=1}^{n d}\left\{N^{i}\right\}$ the set of nodes belonging to the carpooling network which has at most $N n$ nodes. If $N^{i}$ belongs to the $V^{k}$ itinerary Then $I N V\left[N^{i} ; V^{k}\right]=1$ Else $I N V\left[N^{i} ; V^{k}\right]=X$ (see the example in Table 4).

Thanks to the INV matrix, we can identify all the transit nodes between vehicles. For example: If $((I N V) \stackrel{i}{i} ; V$ $\left.{ }^{k}=1\right)$ and $\left.\left(I N V\left[N^{i} ; V^{k^{\prime}}\right]=1\right)\right)$ Then $N^{i}$ can be a transit node between $V^{k}$ and $V^{k^{\prime}}$.

In the following, we define some algorithms used by the MTSA that employ the data modeling matrices of possible assignments vehicle/passenger ( $D V M$ and $T V M)$ and the matrix of interconnected nodes between vehicles $(I N V)$.

Table 4: Matrix INV of Interconnected Nodes between Vehicles
\begin{tabular}{|c|c|c|c|c|c|c|c|}
\hline$N^{i} / V^{k}$ & $V^{1}$ & $V^{2}$ & & $V^{k}$ & & $V^{m} 1$ & $V^{m}$ \\
\hline$N^{1}$ & 1 & 1 & & $\mathrm{X}$ & & $\mathrm{X}$ & 1 \\
\hline$N^{2}$ & $\mathrm{X}$ & 1 & & 1 & & $\mathrm{X}$ & $\mathrm{X}$ \\
\hline$\vdots$ & $\vdots$ & $\vdots$ & & $\vdots$ & $\vdots$ & $\vdots$ & $\vdots$ \\
$N^{l}$ & $\cdot$ & $\cdot$ & & $\cdot$ & $\cdot$ & $\cdot$ & $\cdot$ \\
$\vdots$ & $\vdots$ & $:$ & $:$ & $:$ & & $\vdots$ & $\vdots$ \\
$\cdot$ & $\cdot$ & $\cdot$ & $\cdot$ & $\cdot$ & & $\cdot$ & $\cdot$ \\
\hline$N N n 1$ & 1 & $\mathrm{X}$ & & $\mathrm{X}$ & & 1 & $\mathrm{X}$ \\
\hline$N N n$ & $\mathrm{X}$ & $\mathrm{X}$ & & 1 & & 1 & $\mathrm{X}$ \\
\hline
\end{tabular}

\subsubsection{RAVPA: Random Assignment Vehicle/Passenger Algorithm}

At each iteration of the Random Assignment Vehicle/Passenger Algorithm $(R A V P A)$, each passenger $P^{l}$ is randomly assigned to one vehicle from origin node and to one vehicle to reach his final destination node taking into account the vehicle capacity constraint and the data contained in the matrix DVM and the matrix TVM.

\subsubsection{SCAlgo: Solution Construction Algorithm}

Based on the information provided by the matrices of possible assignments and the vehicles' capacities, a possible solution is generated by a random assignment between raiders and vehicles. The algorithm starts by calling $R A V P A$ to assign each passenger $P^{l}$ to a vehicle $V^{k}$ that will take him from its origin and to a vehicle $V^{k^{\prime}}$ that will transport him from a potential transfer node, while respecting the vehicle capacity constraint. In the case where the passenger is assigned to two different vehicles, SCAlgo verifies in INV if the two vehicles 
have a common intermediate destination to ensure the transfer of the passenger and checks if the time synchronization (constraint (21)) is satisfied. If this is not the case, the triplet $f P^{l} ; V^{k} ; V^{k^{\prime}} g$ will be considered as tabu and SCAlgo saves it in the Tabu List.

As shown, the Solution Construction Algorithm (SCAlgo) consists in browsing several matrices, while ver-ifying certain conditions. According to (4) and the references cited therein, we can consider our SCAlgo as a polynomial algorithm whose complexity is equal to $O\left(n^{3}\right)$.

\subsubsection{Tabu List}

The Tabu List is the feature characterizing the classical T SA. It records the Moves encountered in the past to prevent cycling searches and entrapment in local optima. In our particular case and in order to avoid returning to the local optimum already visited, the Tabu Move is defined as the prohibited transfer between

vehicles $V^{k}$ and $V^{k^{\prime}}$ for the passenger $P^{l}$. Then, the triplet $\left\{P^{l} ; V^{k} ; V^{k^{\prime}}\right\}$ is considered as forbidden and it is saved in the Tabu List.

\subsubsection{Neighborhood construction}

Starting from a current solution, the TSA explores its neighborhood to reach a better solution. To achieve this goal, the algorithm operates with a 'Move' mechanism, allowing movement from a current solution towards another one. In our purposes, we develop a neighborhood construction algorithm that considers the elements of the Tabu List $\left\{P^{l} ; V^{k} ; V^{k^{\prime}}\right\}$ and reassigns the unallocated riders to other vehicles. In the case where the Tabu List is empty, an element is randomly chosen and a new reallocation is proposed, to avoid trapping by a local optimum. Concerning the complexity, the neighborhood construction algorithm has to browse a list. Then, for each element (unallocated passenger), it cheeks the DVM and TVM to find new assignment. Based on (4), we consider the neighborhood construction algorithm as a polynomial algorithm with a complexity $O(n)$. 
Algorithm 1 : SCAlgo: Solution Construction Algorithm (INV, DVM, TVM, CT, MCS, Tabu List)

Require: $I N V, D V M$; $T V M$; $C T$ : Capacity Table, $M D$ : Matrix in which each element $D^{k}{ }_{i}$ is the departure time of $V^{k}$ on $N^{i}, M A$ : Matrix in which each element $A^{k}{ }_{i}$ is the arrival time of $V^{k}$ on $N^{i}$, Tabu List: The set of triplet of $\left(P^{l} ; V^{x} ; V^{y}\right) / /$ Forbidden Transfer

Ensure: $T D A$ : Table of Departure Assignment, $T$ TA: Table of Transit Assignment, CT, MCS: Matrix of Current Solution, Tabu_List

\section{Treatment}

1: RAVPA $(D V M, T D A, C T)$

2: RAVPA (TV $M, T T A, C T)$

3: for all passengers $P^{l}$ do

4: $\quad V^{x} \leftarrow T D A[P]$

5: $\quad V^{y} \leftarrow T T A\left[P^{l}\right]$

6: $\quad$ if $\left(V^{x}=/ 0\right)$ and $\left(V^{y}=/ 0\right)$ then

7: $\quad$ if $\left(V^{x}=V^{y}\right)$ then

8: $\quad \operatorname{MCS}\left[P^{l} ; V^{x}\right]=1$

9: $\quad P^{l} . T N=0 / / T N$ : Transit Node

10: $\quad P^{l} . V^{\text {departure }}=P^{l} \cdot V^{\text {transit }}=V^{x}$

11: $\quad$ else

12: for all node $N^{i}$ belongs to the Carpooling Network do

13: $\quad$ if $\left(I N V\left[N^{i} ; V^{x}\right]=I N V\left[N^{i} ; V^{y}\right]=1\right)$ and $\left(A^{x}{ }_{i} \quad D^{y}{ }_{i}\right.$ then

14: $\quad$ if Time Synchronization is satisfied between $V^{x}$ and $V^{y}$ then

15: $\quad$ if $(C T[x]>0)$ and $\left(C T[y] \prec L^{y}\right)$ then

16: $\quad \operatorname{MCS}\left[P^{l} ; V^{x}\right]=1$

17: $\quad \operatorname{MCS}\left[P^{l} ; V^{y}\right]=2$

18: $\quad C T[x] \leftarrow C T[x]-1$

19: $\quad C T[y] \leftarrow C T[y]+1$

20: $\quad P^{l} \cdot T N=N^{i}$

21: $\quad P^{l} \cdot V^{\text {departure }}=V^{x}$

22: $\quad P^{l}: V^{\text {transit }}=V^{y}$

23: $\quad$ Break

24: $\quad$ end if

25: $\quad$ end if

26: $\quad$ end if

27: $\quad$ end for

28: $\quad$ if $\left(N^{i}=N^{n d}\right)$ and $\left(I N V\left[N^{i} ; V^{x}\right]=I N V\left[N^{i} ; V^{y}\right]\right)$ then

29: $\quad \operatorname{MCS}\left[P^{l} ; V^{x}\right]=\operatorname{MCS}\left[P^{l} ; V^{y}\right]=-1$

30: $\quad$ Tabu List $\leftarrow$ Tabu List $+\{(\mathrm{Pl}, \mathrm{Vx}, \mathrm{Vy})\}$

31: $\quad$ end if

32: $\quad$ end if

33: end if

34: end for 


\subsubsection{Aspiration Criterion}

Even if there is no risk of cycling, tabus may cause a slowdown or even a stagnation of the search process. To deal with this issue, a so-called aspiration criterion is used to revoke Tabus (48). According to Gendreau et al., in classic Tabu Search implementations, the aspiration criterion allows a tabu Move to be feasible if it permit to achieve a non-visited solution with a better performance score than that of the best solution so far known (48). In our case, we risk that the Tabu List forbid some worthy or interesting matching (vehicle/passenger) which can lead to a better solution than the current one. Our original aspiration criterion consists of authorizing forbidden assignments if they are judged interesting. In other words, the aspiration criterion is establish by realizing a detour to serve the riders (Figure 4). This increases the flexibility of the system and improves users satisfaction. The Aspiration Algorithm starts by choosing randomly a triplet $\left\{P^{l}\right.$;

$\left.V^{k} ; V^{k^{\prime}}\right\}$ from the Tabu List. By randomly choosing between tow options, algorithm proposes to $V^{k}$ or $V^{k^{\prime}}$ to make a detour, provided that the detour distance does not exceed the Tolerated Detour Distance $T D D$.

\subsubsection{MCMA global algorithm}

The MCMA global algorithm begins by calling the SCAlgo to generating initial assignments. Once the initial solution is constructed, MCMA evaluates it using the Choquet Integral approach, which is detailed in the subsequent subsection. Then, MCMA calls the neighborhood search algorithm to iteratively move from one potential solution to an improved solution. If the current solution has a better score, compared to the previous one, it will be temporarily considered as the best solution and MCMA will explore its neighborhood, and so on until a stopping criterion is met.

Concerning the terminating conditions: we define two stopped conditions, the first one concerns the the achievement of the maximum number of iterations, while the second condition concerns the quality of the current solution. In other words, if the current best solution is not improving over time, the algorithm is stopped.

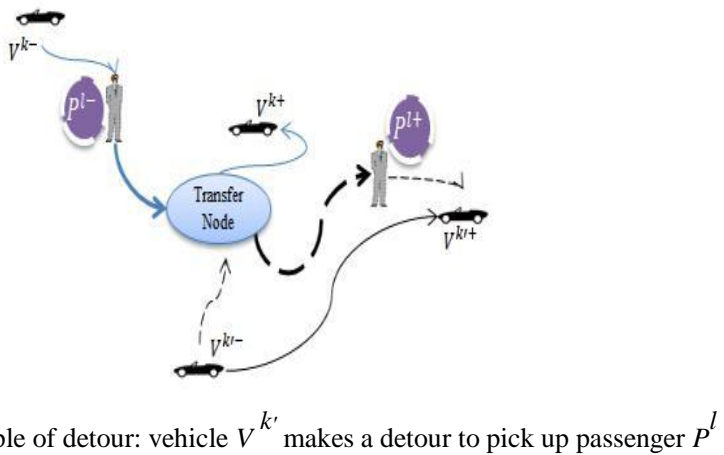

Figure 4: Example of detour: vehicle $V^{k^{\prime}}$ makes a detour to pick up passenger $P^{l}$ 


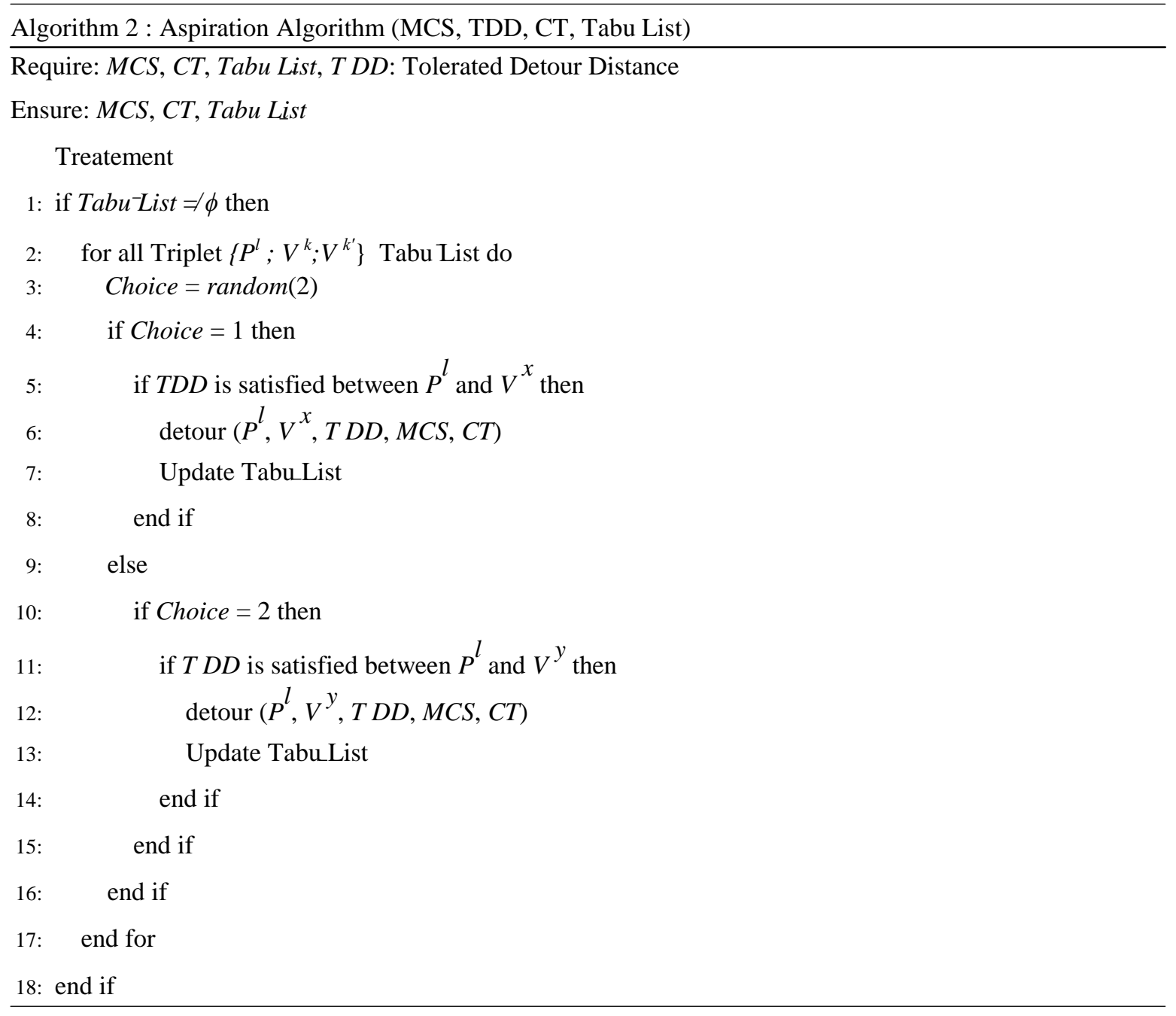


Figure 5 illustrates the flowchart of our global Tabu Search Algorithm, in which we have included the solution construction algorithm, neighborhood algorithm and the aspiration method. We denote $M C S$ the Matrix of the Current Solution and MBS the Matrix of Best Solution.

As consisting of different sub-algorithms with a polynomial complexity and that run sequentially, our multi-criterion metaheuristic algorithm is a polynomial algorithm.

To summarize, our Tabu Search based metaheuristic differs from the classical T SA techniques since it is characterized by:

- Dynamic dimensioning where new passengers and vehicles continuously enter and leave the system. Then, the size of data to be processed remains reasonable,

- Global algorithm with a polynomial complexity,

- Aspiration criterion that enables the research process to move from a local optimum and to improve subsequently the generated assignments,

- Defined stopping criterion.

Thanks to these peculiarities, our Tabu search Algorithm converges towards a valid and good ridematching solution within a reasonable time.

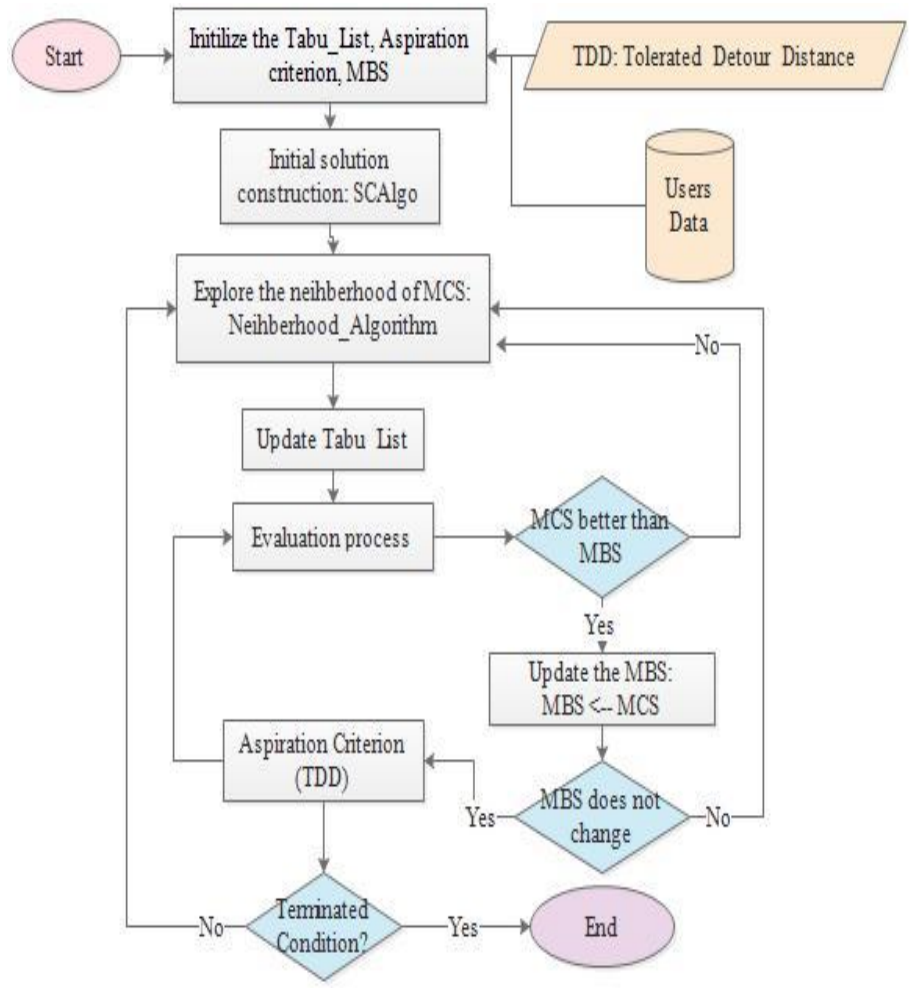

Figure 5: The multi-criterion metaheuristic algorithm 


\subsubsection{Perturbations and their impact on solutions feasibility}

The consideration of disturbances impacting the feasibility of the proposed solutions becomes necessary in a dynamic transport system. In our case, we define three classes of disturbances:

1. Unexpected detour due the the traffic overcrowd problems or the inaccessibility of a section or road.

2. A scheduled transfer that did not take place.

3. A Car breakdown causing the drop off of the passengers.

Thanks to the robustness of our algorithm, the latter modifies the solution to make it feasible while respecting the problem's constraints. However, the handling of this procedure can degrade certain criteria and subsequently influence the service's quality. For example, in the first class of perturbations, making an additional detour can considerably increase the total route time criteria of the concerned passengers in order to achieve the final destination. Regarding the other two classes of disturbances, passengers must get off cars. Thanks to dynamic traceability, our system will be notified of the current position of these passengers and updates their requests with their new position. Thus, the algorithm proposes new feasible solution for these passengers to arrive at their final destination.

\subsection{Solution evaluation process: Aggregation approach based on the Choquet Integral}

In this sub-section we detail the multi-criterion optimization problem and consider the search space exploration to find the best feasible solutions for the proposed DyCOS. The question which arises at this stage of our work is the following: how to get a thorough evaluation of our solution?

As already indicated, the evaluation process follows a multi-criterion approach that focus on optimizing the waiting time, the route time and the accumulated delay on arriving at the final destination. Moreover, our approach takes into consideration the economic criterion by minimizing travel costs and the environmental aspect by optimizing the gain in terms of $\mathrm{CO}_{2}$ realized thanks to carpooling.

One of the popular aggregation methods is the classical weighted arithmetic method. A limitation of this approach, however, is that it does not reflect the interactions among criteria (50). To overcome this drawback, an extended version of the weighted arithmetic operator called the discr ete Choquet integral is developed by (53). In addition to the weighting, the Choquet integral operator takes into consideration the interaction and the compensation between different criteria.

In fact, we notice that our criteria do not have the same importance for users. For example, it is widely acknowledged that for a passenger reaching his final destination without delay is more important than having a good gain of $\mathrm{CO}_{2}$. In addition, according to our mathematical formulation, minimizing travel time (TRT) in-volves minimizing the distance traveled which therefore has an effect on the amount of $\mathrm{CO}_{2}$ emitted. Therefore, we find that both criteria ( $T R T$ and $T E G$ ) are not fully independent and they interact among them. Psychologi-cally speaking, for a passenger, he prefers to reduce the waiting time ( $T W T \searrow$ ), especially when

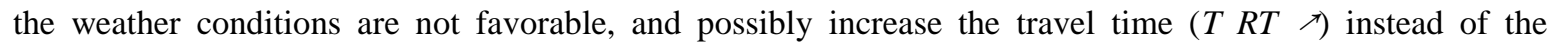
opposite. Using the Choquet Integral operator, this psychological aspect is taken into account by the compensation effect. In this case, the Choquet Integral evaluation can provide a good score. 
To calculate the Choquet score, we have to determine partial scores of criteria. To this aim, we apply the fuzzification method proposed in (66) and in (14) within the framework of disrupted urban transport regulation, to make criteria homogeneous and assessed on the same scale. The proposed fuzzy multi-criterion evaluation procedure follows the steps described above:

- $1^{\text {st }}$ step: Lower-bound determination

To reduce the search space and to characterize the feasible solution limits, we determine a lower-bound for each criterion. In our situation, the lower-bound values represent the five optimization criteria considering the normal traffic conditions.

- $\quad 2^{\text {nd }}$ step: Fuzzification

In order to erase the influence of the difference between the units of different criteria, a fuzzy logic application is adopted (30) (50). Then, the quality of each solution is measured by the combination of the different scores of criteria. These scores are homogeneous and without dimension, and their values lie between 0 and 1 .

- $\quad 3^{r d}$ step: Fuzzy evaluation

It is widely acknowledged that the classical operators, such as the Ordered Weighted Averaging Operators $(O W A)$, used to aggregate criteria suffer from some drawbacks. Although these operators are simple to interpret, they are not able to consider the interaction between criteria in an understandable manner. The fuzzy integrals in general and particularly that of Choquet allow overcoming this disadvantage and representing this kind of behavior perfectly (28). As we can not detail all the theoretical aspects of the Choquet Integral, we present, in the following, the main concepts used for our evaluation process. However, it is possible to consult (28) for more theoretical aspects.

Definition 1: A fuzzy measure $m$ on $N$ (the set of criteria) is a function $m: P(N) ![0 ; 1]$, satisfying the two following conditions:

$$
\mu(\phi)=0
$$

$$
A C B C N \Rightarrow \mu(A) \leq \mu(B)
$$

Depending on the aggregation issue, $m(A)$ is defined as the importance or power of the coalition $A$, where A presents the group of criteria.

Definition 2:Let $\mu$ be a fuzzy measure on $N$ and $a=\left(a_{1} ; \quad ; a_{n c}\right)$ be the vector of criteria. The Choquet integral $C \mu$ with respect to $\mu$ is determined by:

$$
C \mu\left(a_{1 . .} ; a_{n c}\right)=\sum(a i-a i-1) \mu\left(i, . . n_{c}\right)
$$

Where $a_{0}=1$ and $a_{1} \leq \ldots \leq a_{n c}$.

Grabisch et al. states that "A difficulty which has slowed down the application of fuzzy measures is its exponential complexity, since one has to define a real number for each subset of the set of criteria, and also 
to find a mean of evaluating these numbers, either by expert elicitation or by optimization" (28). On this basis, the case of a 2-additive fuzzy measure is notably interesting. In fact, it is more appropriate to model the preference and the interaction between two criteria than between three or more (28). Then, in case of 2additive measurements, the Choquet integral formulation, using interaction index for $a=\left(a_{1} ; ; a_{n c}\right)$, is determined as follows:

$$
C_{\mu}(a)=\sum_{I_{i j} \mathrm{f} 0}\left(a_{i} \wedge a_{j}\right) I_{i j}+\sum_{I_{i j} \mathrm{p} 0}\left(a_{i} \vee a_{j}\right)\left|I_{i j}\right|+\sum_{i=1}^{n} a_{i}\left(I_{i}-\frac{1}{2} \sum_{j \neq i}\left|I_{i j}\right|\right)
$$

Where $a_{i} \in[0 ; 1]$ is the numerical score of criterion $i, \mathrm{~L}$ and $n$ denote min and max, respectively.

It is clear that the Choquet integral for 2 -additive measurements is a combination of a conjunctive, a disjunctive and an additive part, indicating respectively the positive interaction indices, negative interaction and the Shapley value. This indicates clearly the meaning of $I_{i j}$ in the framework of the Choquet integral. According to Ould Sidi et al. in (64):

1. "A positive $I_{i j}$ indicates a conjunctive behaviour between $i$ and $j$. This indicates that the simultaneous satisfaction of criteria $i$ and $j$ has a significant effect on the overall score, but a unilateral satisfaction has no effect. 
2. A negative $I_{i j}$ means a disjunctive behaviour, which implies that the satisfaction of either $i$ or $j$ is adequate to have a significant effect on the overall score.

3. The Shapley value $I_{i}$ acts as a weight vector in a weighted arithmetic mean, where $\sum I i=1$. This corresponds to the linear part of Choquet integral."

- 4 th step: interaction index and Shapley values determination

This step involves calculating the Shapley values and measure the interaction index between criteria (coefficients of Choquet integral) necessary to determine the global score of each generated solution. Several methods were developed for the determination of these parameters (see (28)). However, in the usual case, the decision-maker is supposed to be capable of giving interaction between only two criteria and determining the Shapley values by ordering criteria. For the sake of simplicity, we assume that we are in such a case and suppose that the system manager can express, quantitatively or qualitatively, his preferences and he can determine the interactions index for instance as follows:

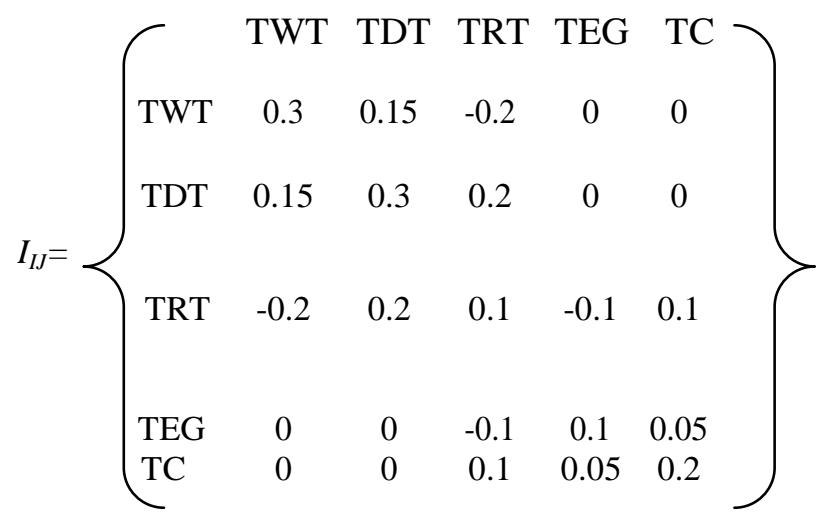

It is interesting to note that the diagonal values indicate the importance of each criterion where $\left(I_{i i}=I_{i}\right)$, while the rest presents the interaction index $\left(I_{i j}\right)$.

Thanks to the use of the Choquet integral evaluating method, we can both explore the convex hull and the concave hull of the solutions space. In fact, the Choquet Integral integrates the the weighted arithmetic method $\left(I_{i}\right.$ acts as a weight vector in a weighted arithmetic mean, where $\left.\sum I_{i}=1\right)$ generating solutions that are located in the convex hull of the solutions space. In addition, taking into account the conjunctive and the disjunctive behaviors between the optimization criteria promotes complementarity between these criteria and avoid redundancy. Moreover, the integration of these interactions allows us to explore Pareto solutions that located in the concave hull of the solutions space.

\section{Simulation results}

\subsection{The simulation environment}


To highlight the merits of optimization for ridesharing management and assess the feasibility of dynamic carpooling with detour and transfer concepts, we define a simulation environment that considers a dynamic carpooling service in a limited geographic area of the Lille metropolitan region in France, known by its road congestion.

The flexibility of the carpooling service is shown in this simulation environment by ensuring the transporta-tion of passengers during a disruption of the urban transport network. Indeed, thanks to this benchmark dynamic ridesharing can be shown to be an emerging mode of transport complementary to the existing multi-modal trans-port network. The goal of our simulation case is to answer the following two questions:

- How many rideshare vehicles are needed to absorb disruption?

- Thanks to the carpooling system, how many riders reach their destinations?

We consider a disturbance, caused by a technical problem, affecting the Metro Line 1 and the bus line 44 between 7 a.m. and 10 a.m. (Figure 6). The aim is to propose a carpooling service allowing the transport of passengers between the different stations affected by the disturbance.

We present here a realistic scenario composed of 20 requests corresponding to 36 passengers with different origins and destinations, that present the network stations. Each rider has to express his preferred departure and arrival times and the number of passengers who will travel with him. The earliest departure among the 20 requests is $6: 55$ a.m. and the latest arrival time is 9:45 a.m. Table 5 details the requests data for the emitted requests. In order to limit the disruption's evolution, it is indispensable to determine the spacetime horizon of the disruption by identifying the set of stations and vehicles concerned by the detected disruption (65). In our case, we have to identify the set of carpooling vehicles operating in the surrounding of the yellow line and the green line.

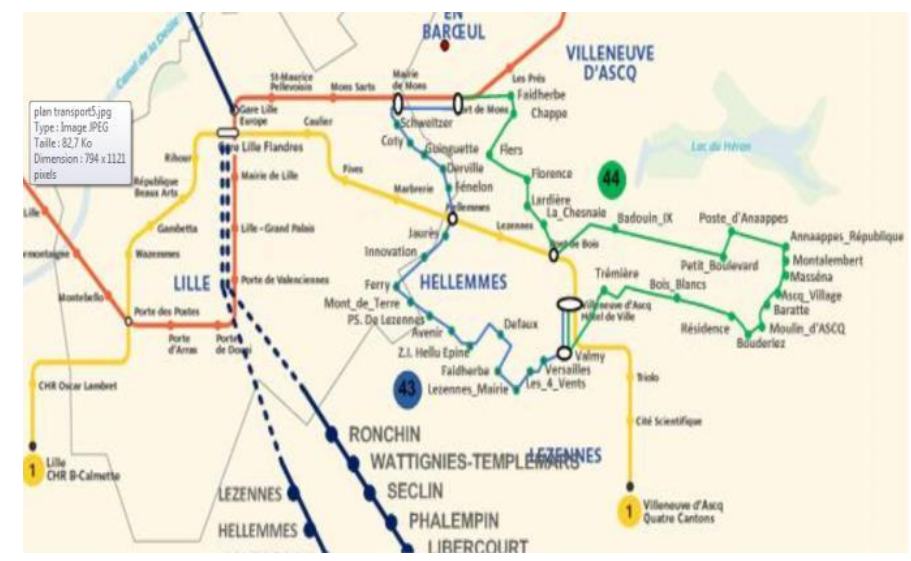

Figure 6: Lille's transportation network 
Table 5: Requests data

\begin{tabular}{|c|c|c|c|c|c|}
\hline$R^{l}$ & $R^{l}$ & $R^{l}$ & $d^{l}$ & $a^{l}$ & $Q^{l}$ \\
\hline$R^{1}$ & 4 Cantons & Republique & $06: 55$ & $07: 15$ & 1 \\
$R^{2}$ & 4 Cantons & Marbrerie & $08: 10$ & $08: 25$ & 2 \\
$R^{3}$ & Marbrerie & Republique & $08: 20$ & $08: 30$ & 2 \\
$R^{4}$ & HDV & Lezennes & $08: 40$ & $08: 50$ & 1 \\
$R^{5}$ & Gambetta & Pont de Bois & $07: 30$ & $08: 00$ & 1 \\
$R^{6}$ & Gambetta & Pont de Bois & $07: 25$ & $07: 55$ & 2 \\
$R^{7}$ & Gambetta & 4 Cantons & $07: 30$ & $07: 55$ & 2 \\
$R^{8}$ & Pont de Bois & 4 Cantons & $07: 40$ & $08: 00$ & 1 \\
$R^{9}$ & Wazemmes & HDV & $08: 00$ & $08: 20$ & 3 \\
$R^{10}$ & Moulin d'ASCQ & Poste d'Anaappes & $07: 35$ & $08: 00$ & 4 \\
$R^{11}$ & Pont de Bois & Florence & $08: 00$ & $08: 15$ & 2 \\
$R^{12}$ & Poste d'Anaappes & Lardiere & $07: 15$ & $07: 30$ & 2 \\
$R^{13}$ & Poste d'Anaappes & Lille Flandres & $07: 15$ & $08: 00$ & 2 \\
$R^{14}$ & Baratte & 4 Cantons & $08: 00$ & $09: 00$ & 2 \\
$R^{15}$ & Baratte & Lezennes & $08: 10$ & $08: 45$ & 2 \\
$R^{16}$ & Cite Scientifique & Residence & $09: 05$ & $09: 30$ & 2 \\
$R^{17}$ & Triolo & Badouin IX & $09: 10$ & $09: 40$ & 2 \\
$R^{18}$ & Ascq Village & 4 Cantons & $08: 50$ & $09: 35$ & 1 \\
$R^{19}$ & Badouin IX & Cite Scientifique & $09: 40$ & $09: 45$ & 2 \\
$R^{20}$ & Lardiere & Porte des postes & $09: 25$ & $09: 45$ & 2 \\
\hline
\end{tabular}

\subsection{Numerical results and discussion}

To illustrate the effectiveness and performance of our system, we present four instances according to the number of vehicles in the carpooling network: 5, 10, 15 and 20 vehicles. Given that the carpooling network is limited and time windows are close, drivers can cooperate together to serve the maximum of passengers' request.

We note that $D y C O S$ is developed with the JAVA language as a connecting module to a geo-localization plat-form called Cartocom (Car). This platform permit to locate drivers and riders in real-time and to communicate with them by using their mobile phones. In our case, Cartocom is used to visualize the vehicules' itineraries generated by our $D y \operatorname{COS}$ (Figure 7). 
Table 6: Test scenarios

\begin{tabular}{|c|c|c|c|c|c|c|}
\hline Instances & \multicolumn{3}{|c|}{ Instance 1 : 5vehicles } & \multicolumn{3}{|c|}{ Instance $2: 10$ vehicles } \\
\hline Scenarios & Scenario 1 & Scenario 2 & Scenario 3 & Scenario 1 & Scenario 2 & Scenario 3 \\
\hline TWT & 0,51 & 0,43 & 0,63 & 0,57 & 0,53 & 0,52 \\
\hline TRT & 0,48 & 0,78 & 0,91 & 0,89 & 0,48 & 0,81 \\
\hline TDT & 0,66 & 0,56 & 0,72 & 0,56 & 0,44 & 0,62 \\
\hline TEG & 0,77 & 0,67 & 0,53 & 0,47 & 0,76 & 0,43 \\
\hline $\mathrm{TC}$ & 0,45 & 0,71 & 0,69 & 0,78 & 0,46 & 0,69 \\
\hline Overall Score & 0,921 & 0,89 & 0,968 & 0,62 & 0,77 & 0,82 \\
\hline$\%$ Served requests & $24 \%$ & $28 \%$ & $22 \%$ & $72 \%$ & $59 \%$ & $61 \%$ \\
\hline$\%$ Transfer rate & $8,11 \%$ & $13,10 \%$ & $11,81 \%$ & $34,12 \%$ & $24,3 \%$ & $19,8 \%$ \\
\hline$\%$ Detour rate & $12,71 \%$ & $19,12 \%$ & $13,31 \%$ & $45,44 \%$ & $24,56 \%$ & $36,45 \%$ \\
\hline Computing Time (s) & 8,33 & 11,22 & 10,54 & 17,34 & 16,32 & 16,20 \\
\hline Instances & \multicolumn{3}{|c|}{ Instance $3: 15$ vehicles } & \multicolumn{3}{|c|}{ Instance $4: 20 v e h i c l e s$} \\
\hline Scenarios & Scenario 1 & Scenario 2 & Scenario 3 & Scenario 1 & Scenario 2 & Scenario 3 \\
\hline TWT & 0,80 & 0,57 & 0,52 & 0,48 & 0,64 & 0,58 \\
\hline TRT & 0,76 & 0,78 & 0,81 & 0,49 & 0,58 & 0,75 \\
\hline TDT & 0,45 & 0,54 & 0,62 & 0,45 & 0,54 & 0,62 \\
\hline TEG & 0,57 & 0,77 & 0,43 & 0,67 & 0,77 & 0,44 \\
\hline $\mathrm{TC}$ & 0,73 & 0,56 & 0,79 & 0,73 & 0,46 & 0,78 \\
\hline Overall Score & 0,75 & 0,69 & 0,72 & 0,63 & 0,68 & 0,66 \\
\hline$\%$ served requests & $68 \%$ & $69 \%$ & $71 \%$ & $78 \%$ & $91 \%$ & $85 \%$ \\
\hline$\%$ Transfer rate & $18,65 \%$ & $19,22 \%$ & $18,44 \%$ & $15,33 \%$ & $21,23 \%$ & $18,22 \%$ \\
\hline$\%$ Detour rate & $23,34 \%$ & $25,45 \%$ & $28,33 \%$ & $14,23 \%$ & $13,43 \%$ & $13,55 \%$ \\
\hline Computing Time (s) & 20,31 & 23,12 & 21,56 & 30,14 & 32,54 & 31,10 \\
\hline
\end{tabular}

The performance results of our approach are presented in Table 6. The table presents three scenarios for each instance: rows 1 to 6 indicate partial score values for different criteria and the overall Choquet score for the best carpooling solution generated by the MCMA for each scenario. We note that the best solution for each instance corresponds to the solution having the lowest overall Choquet score. Row 7 indicates the percentage of served riders' requests. Among the served requests, row 8 and 9 show respectively the percentage of requests which need transfer and detour to reach their final destination. By comparing the different instances, the authorization of detour and transfer processes can improve the values of overall Choquet scores and the number of served requests over the increase in the number of carpooling vehicles. This means that our algorithms is able to make good decisions that are crucial for managing the carpooling matching using a minimum of resources (vehicles).

The main goal of our approach is to increase the number of served riders' requests with a minimum number of carpooling vehicles thanks to the best management of detour and transfer options. However, the 
use of transfer and detour processes may increase the waiting time for carpooling users. Ideally, in the transfer case, the second vehicle arrives at the transfer node exactly when the first vehicle drops off the rider. Unfortunately, our MCMA is an NP-Hard problem, hence, the optimal scenario is difficult to obtain. Therefore, we try to generate a good solution in which, the first one to arrive has to wait for the arrival of the second one. In the literature, it is remarked that "if a vehicle (driver) is waiting for some rider at some transfer point, then the waiting time will be multiplied by the number of the riders already in the vehicle" (31). However, in our approach, in this case, the multiplication of the waiting time by the riders already in the vehicle will be added to their route time $T R T$. This finding is reflected in the compensation between the two criteria $T W T$ and $T R T$ promoting the psychological criterion as already mentioned in the 4.2 section.

Table 6 shows the scores of criteria and the rate of served riders' requests under different numbers of carpooling vehicles. Under the best solution generated by the Scenario 1 for 10 vehicles, we notice that the idea of the authorization of detour and transfer processes add significant improvement. However, the increase in the number of vehicles in circulation on the carpooling network does not necessarily imply a rise in the rate of the served requests. This observation has been reported in Table 6 , for example, by comparing the percentage of served requests in (Instance 2, Scenario 1: 72\% ) and in (Instance 3, Scenario 1: 68\%).

In addition, by analyzing the results in Table IV, we note that in solution 1 (Instance 2, Scenario 1), the effect of compensation between the two criteria TWT (0.57) and TRT (0.89) has not degraded the overall score (0.62) compared to solution 2 (Instance 4, Scenario 1) in which we found almost the same overall score (0.63) but with different scores of TWT (0.48) and TRT (0.49). This result means that the participants show more tolerance with respect to the travel time criterion and this enables the use of Choquet Integral evaluation.

Moreover, in solution 1 , the transfer rate $(34,12 \%)$ and detour rate $(45,44 \%)$ are more important than the transfer rate $(15,33 \%)$ and detour rate $(14,23 \%)$ in solution 2 with almost the same quality of the overall Choquet scores and for almost the same number of served riders' requests. This gives evidence that the idea of authorizing detour and transfer processes could be helpful to decrease the number of involved carpooling vehicles without effect on the overall score.

Table 6 also indicates the computing time of $M C M A$ for the different simulation instants. It is obvious that the growth size of search space increases the temporal complexity.

Figure 7 shows an example of carpooling network which belongs to the disturbance zone of the multimodal transport network and presents results of some drivers' trips considering our best solution generated by the MCMA. In this example, we consider two vehicles $V^{1}$ and $V^{2}$ and six riders, four of them are located by the Cartocom platform at the circle $C 1$ and two of them are located at the circle $C 3$. According to the ride matching generated by our $M C M A$, the vehicle $V^{1}$ arrives at the circle $C 1$ to pick up the four riders. Then, drivers meet at the transfer point $(C 2)$ to exchange riders. In this point, $V^{1}$ will drop off tow riders who will be picked up by the vehicle $V^{2}$ thanks to the transfer process. After that, the vehicle $V^{2}$ continues its itinerary and makes a detour at the $C 3$ circle to pick up the two other passengers, triggering off the aspiration concept of our Tabu algorithm. 
In order to evaluate the performance of our approach, especially compared to other ridematching methods, we draw comparisons between our MCMA and a well knowen algorithm named FCGA (32) in regard to the number of served riders and vehicles involved in the carpooling service. The objective is to demonstrate thec benefit of the transfer process in terms of flexibility and satisfaction client. We denote that FCGA is the abbreviation of Fuzzy Controlled Genetic-based Carpool Problem proposed by Huang et al., in (32). Unlike our $M C M A$, transfer isn't tolerated in FCGA. Although they operate in different simulation environments, $M C M A$ and FCGA have been tested in real-life case studies that consider limited geographical zones.

As shown in Figure 8, thanks to the authorization of transfers and the dynamic establishment of drivers' itineraries, MCMA is able to satisfy a maximum of carpool requests, while involving the minimum of resources (vehicles).

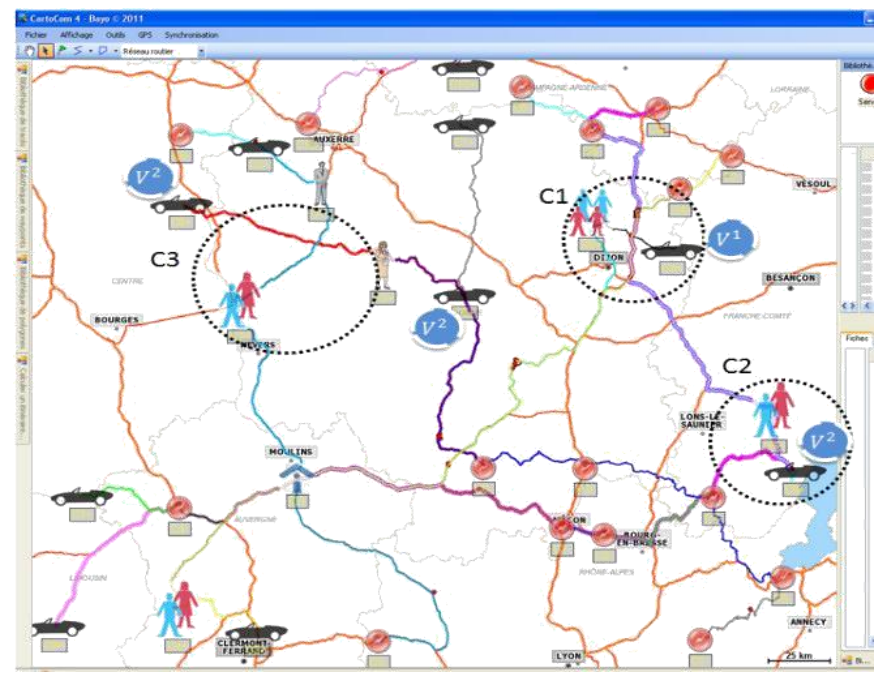

Figure 7: Visualization of the vehicles' itineraries

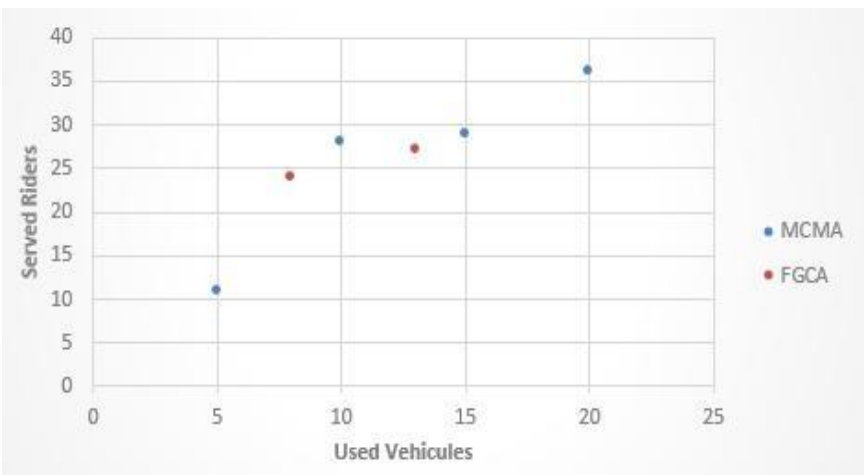

Figure 8: A comparative study between MCMA and FCGA 


\section{Conclusion}

A dynamic carpooling system has to respond readily to unforeseen events and effectively manage users requests, in order to allow its seamless integration with other transport modes and to encourage people to use carpooling. In this study of dynamic ridesharing, we have shown that the use of advanced optimization methods supported by adequate simulation scenarios leads to the definition of a so-called Dynamic Carpooling Optimization System (DyCOS) that has a potential for application in metropolitan areas. Besides allowing travel costs savings and $\mathrm{CO}_{2}$ emission limitation, our dynamic carpooling system helps reducing the traffic congestion and thereby decreasing system-wide travel times. To achieve these objectives, we propose the application of an original Tabu Search optimization method. The developed algorithm generates dynamic and optimal assignments vehicle/passenger. Simulation results on real data from the Lille metropolitan area in northern France show that the idea of the authorization of detour and transfer processes add significant improvement.

However, given the complexity of the dynamic ridematching problem with detour and transfer, the goal is in the first place to verify the feasibility of our resolution approach. In a future step, we plan to implement other methodologies of the literature such as MOPSO, NSGA III, MOGW O, MOWCA, and MOSFS (commonly used algorithms for multi-objective optimization), using our mathematical model and make other comparisons.

In addition, the $D y C O S$ complexity constitutes a handicap for performing an efficient process. Subsequently, this problem can be subdivided into diverse less complex sub-problems. Therefore, in future studies, we intend to investigate the network's subdivision in coordination with a Multi-Agent approach to emphasize the decen-tralized parallel process and to give substance to the distributed aspect of the problem.

Finally, we believe that developing a successful information system in relation to situational factors which affect the relationship between users, such as e.g. attaching attributes to riders and drivers, provides a valuable area of future research. 


\section{References}

[Car] Cartocom : Localisation de vehicules en temps reel avec 1 owasys 22a. [online]. available: http://www.ocmradio.com/uploads/fichetech/67article.pdf

[1] (2004). Wcc: The world carshare consortium, http ://www.cities.worldcarshare.com.

[2] (2009). Hts: National household travel survey. u.s. department of transportation, federal highway administration. [online]. available: http://nhts.ornl.gov.

[3] (2011). Shs: Private transport - car occupancy. [online]. available: http://www.scotland.gov.uk/topics/statistics/browse/transporttravel/trendcaroccupancy.

[4] (2011). wikipedia. [online]. available: https://fr.wikipedia.org/wiki/analyse de la complexite des algorithmes.

[5] Agatz, N., Erera, A., , Savelsbergh, M. W., and Wang, X. (2011). Dynamic ride-sharing: A simulation study in metro atlanta. In Transportation Research Part B: Methodological, volume 45, pages 1450-1464.

[6] Agatz, N., Erera, A., Savelsbergh, M., and Wang, X. (2012). Optimization for dynamic ride-sharing: A review. In European Journal of Operational Research 223 (2), page 295303.

[7] ALONSO-MORA, Javier, S. S.-W.-A. (2017). On-demand high-capacity ride-sharing via dynamic trip-vehicle assignment. In Proceedings of the National Academy of Sciences, volume 114, pages 462-467.

[8] Archetti, C., Speranza, M., and Hertz, A. (2006). A tabu search algorithm for the split delivery vehicle routing problem. In Transporta-tion Science, volume 40, pages 64-73.

[9] Berbeglia, G., Codeau, J., and Laporte, G. (2010). A hybrid tabu search and constraint programming algorithm for dynamic dial a ride problem. In CIRRELT-2010-14.

[10] Blum, C. and Roli, A. (2003). Metaheuristics in combinatorial optimization: Overview and conceptual comparison. In ACM comput-ing surveys (CSUR), volume 35, pages 268-308.

[11] Burris, M. and Winn, J. R. (2006). Slugging in houston: Casual carpool passenger characteristics. In Journal of Public Transportation, page 2340 .

[12] Carli, R., Dotoli, M., Epicoco, N., Angelico, B., and Vinciullo, A. (2015). Automated evaluation of urban traffic congestion using bus as a probe. In Conference on Automation Science and Engineering (IEEE CASE2015), Gothenburg, Sweden, pages 967-972.

[13] Cheikh, S. and Hammadi, S. (2013). The alliance between optimization and multi-agent system for the management of the dynamic carpooling. In Agent and Multi-Agent Systems: Technologies and Applications, Advances in Intelligent Systems and Computing, volume 296, pages 193-202.

[14] Cheikh, S. and S.Hammadi (2013). An optimized evolutionary multi-agent approach for regulation of disrupted urban transport. In International Journal of Modern Engineering Research (IJMER), volume 3, pages 3841-3851.

[15] Cheikh, S. B. and S.Hammadi (2014). Multi-criterion tabu search to solve the dynamic carpooling based on the choquet integral aggregation. In Journal of Traffic and Logistics Engineering, volume 2.

[16] Cheikh, S. B. and S.Hammadi (2016). Multi-hop ridematching optimization problem: Intelligent chromosome agent-driven approach. In Expert Sytems With Applications, volume 62, pages 161-176.

[17] Cheikh, S. B., Tahon, C., and Hammadi, S. (2017). An evolutionary approach to solve the dynamic multi-hop ridematching problem. In Simulation: Transactions of the Society for Modeling and Simulation International, volume 93, pages 3-19.

[18] Chen, C.-M., D.Shallcross, Shih, Y.-C., Wu, Y.-C., Kuo, S.-P., Hsu, Y.-Y., and Holderby, Y. (2011). Smart ride share with flexible route matching. In 13th International Conference on Advanced Communication Technology (ICACT), pages 1506-1510. 
[19] Ching-Fang Liaw, C. C. W. and Bander, J. (1996). A decision support system for the bimodal dial-a-ride problem. In IEEE Transac-tions on Systems, Man, and Cybernetics - Part A: Systems and Humans, volume 26, pages 552-565.

[20] Cordeau, J. and Laporte, G. (2003). A tabu search heuristic for the static multi-vehicle dial-a-ride problem. In Transportation Research, part B, page 579594.

[21] CZIOSKA, Paul, M. D. C. e. S. M. (2017). Gis-based identification and assessment of suitable meeting point locations for ridesharing. In Transportation Research Procedia, volume 22, pages 314-324.

[22] Dimitrakopoulos, G., Demestichas, P., and Koutra, V. (2012). Intelligent management functionality for improving transportation efficiency by means of the car pooling concept. In IEEE Transactions on Intelligent Transportation Systems, volume 13, pages 424-436.

[23] Dotoli, M., Fanti, M., and Meloni, C. (2006). A signal timing plan formulation for urban traffic control. In Control Engineering Practice, volume 14, pages 1297-1311.

[24] Dotoli, M., Hammadi, S., Jeribi, K., Russo, C., and Zgaya, H. (2013). A multi-agent decision support system for optimization of comodal transportation route planning services. In 52nd Conference on Decision and Contro (IEEE CDC), Florence, Italy, pages 911 $-916$.

[25] Fiechter, C. (1994). A parallel tabu search algorithm for large traveling salesman problems. In Discrete Applied Mathematics, volume 51, pages 243-267.

[26] Furuhata, M., Dessouky, M., Ordez, F., Brunet, M., Wang, X., and Koenig, S. (2013). Ridesharing: The state-of-the-art and future directions. In Transportation Research Part B: Methodological, volume 57, page 2846.

[27] Geisberger, R., Luxen, D., Neubauer, S., P.Sanders, and Volker, L. (2009). Fast detour computation for ride sharing. In Clinical Orthopaedics and Related Research, abs/0907.5269.

[28] Grabisch, M. and Roubens, M. (2002). Applications of the choquet integral in multicriteria decision making. fuzzy measures and integrals: theories and applications. In Phisica Verlag, pages 415-434.

[29] Graziotin, D. (2010). An analysis of issues against the adoption of dynamic carpooling. In Free University of Bozen-Bolzano, pages $1-6$.

[30] Hayward, G. and Davidson, V. (2003). Fuzzy logic applications. In Analyst.

[31] Herbawi, W. and Weber, M. (2012). Modeling the multihop ridematching problem with time windows and solving it using genetic algorithms. In IEEE 24th International Conference on Tools with Artificial Intelligence, pages 89-96.

[32] Huang, S., Jiau, M., and Lin, C. (2015). A genetic-algorithm-based approach to solve carpool service problems in cloud computing. In IEEE Transactions on Intelligent Transportation Systems, volume 16, pages 866-876.

[33] HUANG, Can, Z. D. S. Y.-W. (2016). Tabu search for the real-world carpooling problem. In Journal of Combinatorial Optimization, volume 32, pages 492-512.

[34] Jespersen-Groth, J., Potthoff, D., Clausen, J., and D.Huisman (2008). Disruption management in passenger railway transportation. In Computers and Operations Research.

[35] J.F. Cordeau, G. L. (2005). Tabu search heuristics for the vehicle routing problem. In Metaheuristic Optimization via Memory and Evolution Operations Research/Computer Science Interfaces Series, volume 30, pages 145-163.

[36] J.Ferreira, P.Trigo, and P.Filipe (2009). Collaborative car pooling system. In World Academy of Science, Engineering and Technology, volume 54, page 2846.

[37] Khalilpourazari, Soheyl, P. S. H. R.-e. G. A. (2018a). Robust possibilistic programming for multi-item eoq model with defective supply batches: Whale optimization and water cycle algorithms. In Neural Computing and Applications, pages 1-28.

[38] Khalilpourazari, Soheyl et KHALILPOURAZARY, S. (2018b). Optimization of time, cost and surface roughness in grinding process using a robust multi-objective dragonfly algorithm. In Neural Computing and Applications, pages 1-12. 
[39] Khalilpourazari, Soheyl et KHALILPOURAZARY, S. (2018c). Scwoa: an efficient hybrid algorithm for parameter optimization of multi-pass milling process. In Journal of Industrial and Production Engineering, volume 35, pages 35-147.

[40] KHALILPOURAZARI, Soheyl et PASANDIDEH, S. H. R. (2018). Multi-objective optimization of multi-item eoq model with partial backordering and defective batches and stochastic constraints using mowca and mogwo. In Operational Research, pages 1-33.

[41] Khalilpourazari, Soheyl et PASANDIDEH, S. H. R. (2019). Modeling and optimization of multi-item multi-constrained eoq model for growing items. In Knowledge-Based System, volume 164, pages 150-162.

[42] Kleiner, A., Nebel, B., and Ziparo., V. (2011). A mechanism for dynamic ride sharing based on parallel auctions. In In: Proc. of . the 22th International Joint Conference on Artificial Intelligence (IJCAI), Barcelona, Spain, page 266272.

[43] Kris, B., Katrien, R., and Inneke, V. N. (2016). The vehicle routing problem: State of the art classification and review. In Computers Industrial Engineering, volume 99, pages 300-313.

[44] Lalos, P., Korres, A., Datsikas, C., Tombras, G., and Peppas, K. (2009). A framework for dynamic car and taxi pools with the use of positioning systems. In 2009 Computation World: Future Computing, Service Computation, Cognitive, Adaptive, Content, Patterns, pages 385-391.

[45] Lecchini, A., Glover, W., Lygeros, J., and Maciejowski, J. (2006). Monte carlo optimisation for conflict resolution in air traffic control. In IEEE Transactions on Intelligent Transportation Systems, pages 470-482.

[46] Likhachevt, M., Ferguson, D., Gordon, G., Stentz, A., and Thrun, S. (2005). Anytime dynamic a*: An anytime, replanning algorithm.

[47] M. Clemente, M. P. Fanti, G. I. M. N. and Ukovich, W. (2018). A decision support system for user-based vehicle relocation in car sharing systems. In IEEE Transactions on Systems, Man, and Cybernetics: Systems, volume 48, pages 1283-1296.

[48] M. Gendreau, J. P. (2005). Tabu search. In Search Methodologies, Springer, pages 165-186.

[49] M. P. Fanti, A. M. Mangini, G. P. and Ukovich, W. (2017). Fleet sizing for electric car sharing systems in discrete event system frameworks. In IEEE Transactions on Systems, Man, and Cybernetics: Systems.

[50] Mariagrazia, D., Nicola, E., and Marco, F. (2015). A cross-efficiency fuzzy data envelopment analysis technique for performance evaluation of decision making units under uncertainty. In Computers and Industrial Engineering, volume 79, pages 103-114.

[51] M.Bruglieria, D.Ciccarellib, A.Colornia, and A. (2011). Poliunipool: a carpooling system for universities. In Procedia Social and Behavioral Sciences, volume 20, pages 1450-1464.

[52] Mudaliar, D. N. and Modi, N. K. (2013). Unraveling travelling salesman problem by genetic algorithm using m-crossover operator. In Signal Processing Image Processing and Pattern Recognition (ICSIPR), pages 127-130.

[53] Murofushi, T. and Sugeno, M. (1991). A theory of fuzzy measures: Representations, the choquet integral, and null sets. In Journal of Mathematical Analysis and Applications, pages 532-549.

[54] Nagare, D., More, K., Tanwar, N., S.S.Kulkarni, and Gunda, K. C. (2013). Dynamic carpooling application development on android platform. In International Journal of Innovative Technology and Exploring Engineering (IJITEE), volume 2, pages 136-139.

[55] Nanry, W. and Barnes, W. (2000). Solving the pickup and delivery problem with time windows using reactive tabu. In Transportation Research Part B: Methodological, page 107121.

[56] Osman, I. H. and Laporte, G. (1996). Metaheuristics: A bibliography. In Annals of Operations Research, volume 63, pages 513-623.

[57] P.Gruebele (2008). Interactive system for real time dynamic multi-hop carpooling. In Global Transport Knowledge Partnership.

[58] R. Wolfler Calvo, F. De Luigi, P. H. V. (2004). A distributed geographic information system for the daily car pooling problem. In Computers and Operations Research, volume 31, pages 2263-2278.

[59] Schrank, D., Eisele, B., and Lomax, T. (2012). Ttis 2012 urban mobility report powered by inrix traffic data. [online]. available: http://d2dt15nnlpfr0r.cloudfront.net/tti.tamu.edu/documents/mobility-report-2012.pdf. In Texas AM Transportation Institute. 
[60] Sghaier, M., Hammadi, S., Zgaya, H., and Tahon, C. (2011a). A novel approach based on a distributed dynamic graph modeling set up over a subdivision process to deal with distributed optimized real time carpooling requests. In 14th International IEEE Conference on in Intelligent Transportation Systems (ITSC), pages 1311-1316.

[61] Sghaier, M., Hammadi, S., Zgaya, H., and Tahon, C. (2011b). An optimized dynamic carpooling system based on communicating agents operating over a distributed architecture. In 11th International Conference on Intelligent Systems Design and Applications (ISDA), pages 124- 129 .

[62] Shete, A., Bhandare, V., Londhe, L., and Mali, P. (2015). Intelligent carpooling system. In International Journal of Computer Applications, volume 118, pages 26-31.

[63] Shi, L. and Pan, Y. (2005). An efficient search method for job-shop scheduling problems. In IEEE Transactions on Automation Science and Engineering, pages $73-77$.

[64] Sidi, M. M. O., Hayat, S., Hammadi, S., and Borne, P. (2008). A novel approach to developing and evaluating regulation strategies for urban transport disrupted networks. In International Journal of Computer Integrated Manufacturing, pages $480-493$.

[65] Sidi, M. O. (2006). Contribution a l'amelioration des systemes d'aide a la decision dans le domaine du transport. In These de doctorat, Ecole Centrale de Lille.

[66] Sidi, M. O. and Hammadi, S. (2008). Urban transport networks regulation and evaluation: A fuzzy evolutionary approach. In IEEE Transport System, Man, Cybern SMC Part A, page 309318.

[67] Son, T., Hoai, L., Taol, P., and Khadraoui, D. (2012). A distributed algorithm solving multiobjective dynamic car pooling problem. In International Conference on Computer and Information Science (ICCIS), pages 231-236.

[68] S.Seyedabrishami, A.Mamdoohi, A.Barzegar, and S.Hasanpour (2012). Impact of carpooling on fuel saving in urban transportation: Case study of tehran. In Procedia - Social and Behavioral Sciences, volume 54, page 323331.

[69] Wang, X., Dessouky, M., and Ordonez, F. (2015). A pickup and delivery problem for ridesharing considering congestion. In Maney Publishing.

[70] W.Herbawi and M.Weber (2011). Evolutionary multiobjective route planning in dynamic multi-hop ridesharing. In Evolutionary Computation in Combinatorial Optimization, volume 6622, pages 84-95.

[71] XIA, Jizhe, C. K. M. L. W. (2015). A new model for a carpool matching service. In PloS ones, volume 10 , pages $462-467$.

[72] XIAO, Qiang et HE, R.-C. (2017). Carpooling scheme selection for taxi carpooling passengers: a multi-objective model and optimisation algorithm. In Archives of Transport, volume 42.

[73] Xingquan, Z., Murray, C., and Smith, A. (2014). Solving an extended double row layout problem using multiobjective tabu search and linear programming. In IEEE Transactions on in Automation Science and Engineering, volume 11, pages 1122-113. 